\title{
REPRESENTATION THEORY OF PARTIAL RELATION EXTENSIONS
}

\author{
IBRAHIM ASSEM, JUAN CARLOS BUSTAMANTE, JULIE DIONNE, \\ PATRICK LE MEUR, AND DAVID SMITH \\ Dedicated to Claude Cibils for his 60th birthday
}

\begin{abstract}
Let $C$ be a finite dimensional algebra of global dimension at most two. A partial relation extension is any trivial extension of $C$ by a direct summand of its relation $C-C$-bimodule. When $C$ is a tilted algebra, this construction provides an intermediate class of algebras between tilted and cluster tilted algebras. The text investigates the representation theory of partial relation extensions. When $C$ is tilted, any complete slice in the Auslander-Reiten quiver of $C$ embeds as a local slice in the Auslander-Reiten quiver of the partial relation extension. Moreover, a systematic way of producing partial relation extensions is introduced by considering direct sum decompositions of the potential arising from a minimal system of relations of $C$.
\end{abstract}

\section{INTRODUCTION}

Cluster tilted algebras were introduced in 14 and independently in 15 for the $\mathbb{A}$ case, as a by-product of the now extensive theory of cluster algebras of Fomin and Zelevinsky. They have been the subject of many investigations. In particular, it was proved in 2] that a cluster tilted algebra can always be written as the relation extension of a tilted algebra $C$, that is, the trivial extension of $C$ by the so-called relation bimodule $E=\operatorname{Ext}_{C}^{2}(D C, C)$. Tilted algebras have been characterised by the existence of complete slices in their module categories, see, for instance, [6]. It was proven in [4] that any complete slice in the module category of a tilted algebra $C$ embeds in the module category of its relation extension $\widetilde{C}$ as what is called a local slice. However, as seen in [4], the existence of local slices does not characterise cluster tilted algebras, and it was asked there which algebras are characterised by the existence of local slices. Our objective in the present paper is to exhibit another natural class of algebras admitting local slices.

Because cluster tilted algebras are Jacobian algebras of quivers with potential, as shown in [11, we take this context as our starting point. We define the notion of direct sum decomposition of the Keller potential of the relation extension of a triangular algebra $C$ with global dimension at most two. In this case, a direct sum decomposition of the potential associated

2010 Mathematics Subject Classification. Primary 16G10; Secondary 16G70,16E30.

Key words and phrases. representation theory, finite dimensional algebra, partial relation extension, tilted algebra, cluster tilted algebra, cluster category, Auslander-Reiten quiver, local slice, quiver with potential. 
with the relation extension of $C$ induces a direct sum decomposition of the relation bimodule. It is reasonable to expect that the converse statement also holds true. We can prove this converse in two cases where a minimal system of minimal relations is known, namely the cluster tilted algebras with a cyclically oriented quiver of [9], which include all the representationfinite cluster tilted algebras, see [13], and the cluster tilted algebras of type $\widetilde{\mathbb{A}}$ of [1]. Referring to section 1 for the definitions, our first theorem reads as follows.

Theorem 1 (Propositions 1.2.2, 1.3.2 and 1.4.2). Let $C=\mathbb{k} Q / I$ be a triangular algebra of global dimension at most two, and $W$ be the Keller potential of its relation extension associated with a minimal system of relations in $I$. If $W=W^{\prime} \oplus W^{\prime \prime}$ is a direct sum decomposition and $E^{\prime}, E^{\prime \prime}$ are the partial relation bimodules corresponding to $W^{\prime}, W^{\prime \prime}$ respectively, then

$$
E=E^{\prime} \oplus E^{\prime \prime}
$$

as $C-C$-bimodules.

Conversely, if $\widetilde{C}=C \ltimes \operatorname{Ext}_{C}^{2}(D C, C)$ is a cluster tilted algebra with a cyclically oriented quiver or a cluster tilted algebra of type $\widetilde{\mathbb{A}}$ and $E=$ $E^{\prime} \oplus E^{\prime \prime}$ is a direct sum decomposition of $E$ as $C-C$-bimodules, then there exists a direct sum decomposition of the Keller potential

$$
W=W^{\prime} \oplus W^{\prime \prime}
$$

such that $E^{\prime}, E^{\prime \prime}$ are the partial relation bimodules corresponding to $W^{\prime}, W^{\prime \prime}$ respectively.

We then define the class of algebras we are interested in. Let $C$ be a triangular algebra of global dimension at most two, and $E=E^{\prime} \oplus E^{\prime \prime}$ be a direct sum of $C-C$-bimodules, then the algebra $B=C \ltimes E^{\prime}$ is called a partial relation extension of $C$. Because it is easily shown that $\widetilde{C}=B \ltimes E^{\prime \prime}$, partial relation extensions can be thought of as an intermediate class of algebras between tilted and cluster tilted algebras (or more generally, between a triangular algebra of global dimension at most two, and its relation extension). The bound quiver of a partial relation extension is easily computed and we then proceed to study its module category, obtaining the following theorem when the original algebra $C$ is tilted.

Theorem 2. Let $H$ be a hereditary algebra, $\mathcal{C}_{H}$ its cluster category, $T_{H}$ be a tilting $H$-module and $C=\operatorname{End}_{H}(T)$. Then, there exists an ideal $\mathcal{K}$ in the cluster category such that the composition $(-\underset{\widetilde{C}}{\otimes} B) \circ \operatorname{Hom}_{\mathcal{C}_{H}}(T,-): \mathcal{C}_{H} \rightarrow$ $\bmod \widetilde{C} \rightarrow \bmod B$ induces an equivalence

$$
\bmod B \simeq \mathcal{C}_{H} / \mathcal{K}
$$

The ideal $\mathcal{K}$ is characterised by approximations in the cluster category. It is important to observe that, in contrast to what happens for cluster tilted algebras, factoring by $\mathcal{K}$ does not mean simply deleting finitely many objects of $\mathcal{C}_{H}$ : we may have $H$ representation-infinite and $B$ representationfinite. As an easy consequence of our theorem 2, we obtain a full and dense 
functor from the module category of the cluster repetitive algebra of $C$ to $\bmod B$. Returning to our original motivation, we finally prove the following result.

Theorem 3. Let $C$ be a tilted algebra and $A$ be an algebra such that there exist surjective algebra morphisms $\widetilde{C} \rightarrow A \rightarrow C$. Then any complete slice in $\Gamma(\bmod C)$ embeds as a local slice in $\Gamma(\bmod A)$. In particular, partial relation extensions admit local slices.

Notice however that $\mathrm{H}$. Treffinger [19] has obtained a very large class of algebras having local slices, comprising partial relation extensions.

We devote a section of the paper to the proof of each of the stated theorems.

\section{Decomposition of the potential and the Relation Bimodule}

1.1. Decompositions of a potential. Let $(Q, W)$ be a pair consisting of a finite quiver $Q$ and a potential $W$, that is, a linear combination of oriented cycles of $Q$. Define a relation between the (oriented) cycles which appear as summands of $W$ as follows: $\gamma \sim \gamma^{\prime}$ whenever there exists an arrow $\alpha \in Q_{1}$ which is common to both $\gamma$ and $\gamma^{\prime}$. This relation is reflexive and symmetric, let $\approx$ be its transitive closure (that is, the smallest equivalence relation containing it).

Two cycles $\gamma$ and $\gamma^{\prime}$ are called independent if $\gamma \not \approx \gamma^{\prime}$, and dependent if $\gamma \approx \gamma^{\prime}$.

A sum decomposition of the potential

$$
W=W^{\prime}+W^{\prime \prime}
$$

is said to be direct if, whenever $\gamma^{\prime}$ is any cycle in $W^{\prime}$ and $\gamma^{\prime \prime}$ is any cycle in $W^{\prime \prime}$, we have $\gamma^{\prime} \not \gamma^{\prime \prime}$. We denote a direct sum decomposition of the potential as $W=W^{\prime} \oplus W^{\prime \prime}$.

Example 1.1.1.

(a) Let $(Q, W)$ be the quiver

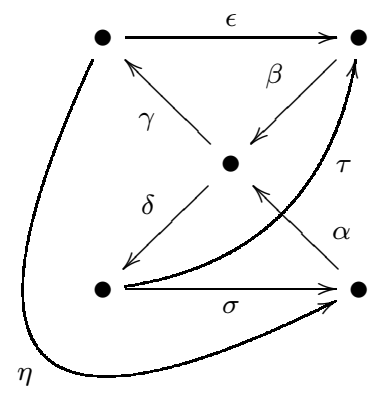

with $W=\beta \gamma \epsilon+\beta \delta \tau+\alpha \gamma \eta+\alpha \delta \sigma$. Here, the four summands of the potential are pairwise dependent. 
(b) Let $(Q, W)$ be the quiver

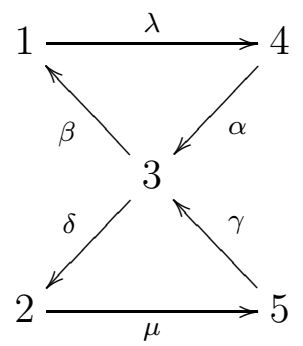

with $W=\alpha \beta \lambda+\gamma \delta \mu$. Here the two cycles $\alpha \beta \lambda$ and $\gamma \delta \mu$ are independent so the decomposition $W=W_{1}+W_{2}$ with $W_{1}=\alpha \beta \lambda$ and $W_{2}=\gamma \delta \mu$ is direct, and $W=W_{1} \oplus W_{2}$.

1.2. Induced decompositions of the relation bimodule. Our objective is to apply the notion of direct sum decompositions of the potential to the study of cluster tilted algebras. We refer the reader to [14] and to 2] for general background on cluster tilted algebras. In particular let $C$ be a triangular algebra of global dimension at most two and consider the $C-C$-bimodule $E=\operatorname{Ext}_{C}^{2}(D C, C)$ equipped with the natural left and right actions of $C$. This bimodule $E$ is called the relation bimodule and the trivial extension algebra $\widetilde{C}=C \ltimes E$ is called the relation extension of $C$. The best known class of relation extensions is provided by the cluster tilted algebras: it is shown in [2, (3.4)] that, if $C$ is a tilted algebra, then $\widetilde{C}$ is cluster tilted, and every cluster tilted algebra arises in this way.

The bound quiver of a relation extension is constructed as follows. Let $C=\mathbb{k} Q / I$ be an admissible presentation of $C$. A subset $R=\left\{\rho_{1}, \ldots, \rho_{t}\right\}$ of $\bigcup_{x, y \in Q_{0}} e_{x} I e_{y}$ is called a system of relations for $C$ if $R$, but no proper subset of $R$, generates $I$ as a two-sided ideal, see [10, (1.2)]. The ordinary quiver $\widetilde{Q}$ of $\widetilde{C}$ has the same vertices as those of $Q$, while the set of arrows in $\widetilde{Q}$ from $x$ to $y$, say, equals the set of arrows in $Q$ from $x$ to $y$, plus, for each relation $\rho \in R \cap e_{y} I e_{x}$, a so-called new arrow $\alpha_{\rho}: x \rightarrow y$, see [2, (2.6)]. Thus $\widetilde{C}$ is not triangular unless $C$ is hereditary and, if $R=\left\{\rho_{1}, \ldots, \rho_{r}\right\}$ is as above, and the new arrow $\alpha_{i}$ corresponds to $\rho_{i}$, then $\alpha_{i} \rho_{i}$ is an oriented cycle in $\widetilde{Q}$. We define the Keller potential (associated with $R$ ) by setting

$$
W=\sum_{i=1}^{t} \alpha_{i} \rho_{i}
$$

Oriented cycles in potentials are, as usual, considered up to cyclic permutations: two potentials are called cyclically equivalent if their difference lies in the linear span of all elements of the form $\gamma_{1} \gamma_{2} \cdots \gamma_{m}-\gamma_{m} \gamma_{1} \cdots \gamma_{m-1}$ where $\gamma_{1} \cdots \gamma_{m}$ is an oriented cycle. For a given arrow $\beta$, the cyclic partial derivative $\partial_{\beta}$ of $W$ is defined on each cyclic summand $\gamma_{1} \cdots \gamma_{m}$ of $W$ by

$$
\partial_{\beta}\left(\gamma_{1} \cdots \gamma_{m}\right)=\sum_{\beta=\gamma_{i}} \gamma_{i+1} \cdots \gamma_{m} \gamma_{1} \cdots \gamma_{i-1}
$$

In particular, cyclic derivatives are invariant under cyclic permutations. The Jacobian algebra $J(\widetilde{Q}, W)$ is the one given by the quiver $\widetilde{Q}$ bound by all 
partial cyclic derivatives $\partial_{\beta} W$ of the Keller potential $W$ with respect to each arrow $\beta \in \widetilde{Q}_{1}$. Then the relation extension $\widetilde{C}$ is isomorphic to $J(\widetilde{Q}, W) / \mathcal{J}$ where $\mathcal{J}$ is the square of the ideal of $J(\widetilde{Q}, W)$ generated by the new arrows, see [5, Lemma 5.2]. If, in particular, $C$ is tilted, so that $\widetilde{C}$ is cluster tilted, then $\widetilde{C} \simeq J(\widetilde{Q}, W)$, see for instance [18].

Setting $\widetilde{C}=\mathbb{k}_{k} \widetilde{Q} / \widetilde{I}$, we recall from [2, (2.4)] that the classes of arrows (modulo $\widetilde{I}$ ) which belong to $\widetilde{Q}_{1} \backslash Q_{1}$ are the generators of the $C-C$-bimodule E.

Before proving the main result of the subsection, we need a technical lemma. We assume that $C$ is a triangular algebra of global dimension at most two, and that $\widetilde{C}$ is its relation extension.

Lemma 1.2.1. With the above notation, consider a partition of the set of new arrows $\widetilde{Q}_{1} \backslash Q_{1}=F^{\prime} \cup F^{\prime \prime}$. Let $E^{\prime}, E^{\prime \prime}$ be the subbimodules of $E$ generated by the classes of the arrows in $F^{\prime}$ and $F^{\prime \prime}$, respectively. If $E^{\prime} \cap E^{\prime \prime} \neq 0$ then there exist oriented cycles $\gamma^{\prime}, \gamma^{\prime \prime}$ in $W$ such that

(1) $\gamma^{\prime}$ has one or two arrows in $\widetilde{Q}_{1} \backslash Q_{1}$, and at least one of them lies in $F^{\prime}$

(2) $\gamma^{\prime \prime}$ has one or two arrows in $\widetilde{Q}_{1} \backslash Q_{1}$, and at least one of them lies in $F^{\prime \prime}$,

(3) $\gamma^{\prime}$ and $\gamma^{\prime \prime}$ have a common arrow,

(4) $\gamma^{\prime}$ has two arrows in $\widetilde{Q}_{1} \backslash Q_{1}$ if and only if so does $\gamma^{\prime \prime}$, in which case $\gamma^{\prime}$ and $\gamma^{\prime \prime}$ have a common arrow in $\widetilde{Q}_{1} \backslash Q_{1}$.

Proof. Let $e$ be a nonzero element in $E^{\prime} \cap E^{\prime \prime}$. There exist paths $u_{1}, \ldots, u_{m}$, $v_{1}, \ldots, v_{n}$ and scalars $\lambda_{1}, \ldots, \lambda_{m}, \mu_{1}, \ldots, \mu_{n}$ satisfying the following conditions

(a) e equals both classes of $\sum_{i} \lambda_{i} u_{i}$ and $\sum_{j} \mu_{j} v_{j}$,

(b) each $u_{i}$ has exactly one arrow from $\widetilde{Q}_{1} \backslash Q_{1}$ and that arrow lies in $F^{\prime}$, we denote this arrow by $\alpha_{i}^{\prime}$,

(c) each $v_{i}$ has exactly one arrow from $\widetilde{Q}_{1} \backslash Q_{1}$ and that arrow lies in $F^{\prime \prime}$, we denote this arrow by $\alpha_{i}^{\prime \prime}$.

Therefore, there exist paths $a_{1}, \ldots, a_{N}, b_{1}, \ldots, b_{N}$, scalars $t_{1}, \ldots, t_{N}$ and arrows $\beta_{1}, \ldots, \beta_{N}$ such that

$$
\sum_{i} \lambda_{i} u_{i}-\sum_{j} \mu_{j} v_{j}=\sum_{\ell} t_{\ell} a_{\ell} \cdot \partial_{\beta_{\ell}} W \cdot b_{\ell} .
$$

In view of condition (a) above and because $e \neq 0$, there exists $\ell$ such that the expression $a_{\ell} \cdot \partial_{\beta_{\ell}} W \cdot b_{\ell}$ contains both $u_{i}$ and $v_{j}$ for some indices $i, j$. Note that neither $\alpha_{i}^{\prime}$ nor $\alpha_{j}^{\prime \prime}$ appears in some $a_{\ell}$ or $b_{\ell}$ for, otherwise, both would appear in $u_{i}$ and $v_{j}$, thus contradicting conditions (b) and (c) above. Hence, there exist oriented cycles $\gamma^{\prime}, \gamma^{\prime \prime}$ that appear in $W$, that contain $\alpha_{i}^{\prime}$ and $\alpha_{j}^{\prime \prime}$, respectively, and that both contain $\beta_{\ell}$.

Since any cycle in $W$ contains at most one arrow from $\widetilde{Q}_{1} \backslash Q_{1}$ it follows that $\gamma^{\prime}$ contains at most two arrows from $\widetilde{Q}_{1} \backslash Q_{1}$ (namely $\alpha_{i}^{\prime} \in F^{\prime}$ and 
possibly $\beta_{\ell}$ ). Whence (1). Assertion (2) follows from similar considerations. Moreover, $\gamma^{\prime}$ and $\gamma^{\prime \prime}$ have the arrow $\beta_{\ell}$ in common. This shows (3) and (4).

In view of the preceding lemma, we define for each direct summand $W^{\prime}$ of the potential $W$ in $\mathbb{k} \widetilde{Q}$ the subbimodule $E^{\prime}$ of $E$ as follows: $E^{\prime}$ is generated by the classes of arrows in $\widetilde{Q}_{1} \backslash Q_{1}$ appearing in a cycle of $W^{\prime}$. We call $E^{\prime}$ the partial relation bimodule corresponding to $W^{\prime}$.

Proposition 1.2.2. Let $W=W^{\prime} \oplus W^{\prime \prime}$ be a direct sum decomposition of the potential. Then $E=E^{\prime} \oplus E^{\prime \prime}$ where $E^{\prime}$ and $E^{\prime \prime}$ are the partial relation bimodules corresponding to $W^{\prime}$ and $W^{\prime \prime}$, respectively.

Proof. Let $F^{\prime}$ and $F^{\prime \prime}$ be the set of arrows in $\widetilde{Q}_{1} \backslash Q_{1}$ appearing in a cycle from $W^{\prime}$ and $W^{\prime \prime}$, respectively. By construction of $W$, the union $F^{\prime} \cup F^{\prime \prime}$ equals $\widetilde{Q}_{1} \backslash Q_{1}$. And because the decomposition $W=W^{\prime}+W^{\prime \prime}$ is direct, $F^{\prime} \cap F^{\prime \prime}=\emptyset$. The preceding lemma therefore applies: because $W=W^{\prime}+W^{\prime \prime}$ is a direct sum decomposition, it entails that $E^{\prime} \cap E^{\prime \prime}=0$. On the other hand $E=E^{\prime}+E^{\prime \prime}$ because $F^{\prime} \cup F^{\prime \prime}=\widetilde{Q}_{1} \backslash Q_{1}$.

It is natural to ask if, conversely, given a direct sum decomposition of the relation bimodule $E=E^{\prime} \oplus E^{\prime \prime}$, one can get a corresponding decomposition of the potential. The next two subsections are devoted to this problem.

In order that the converse process be possible, it seems to be needed that a presentation of the cluster tilted algebra by minimal relations be given by the potential. It is known that this is not always the case, see 9 , Example 4.3]. Recall that, following [13], a minimal relation in a bound quiver $(Q, I)$ is any element of $I$ not lying in $\underline{r} I+I \underline{r}$, where $\underline{r}$ denotes the two-sided ideal of $\mathbb{k} Q$ generated by all the arrows of $Q$. The problem of finding systems of minimal relations for a cluster tilted algebra or, more generally, Jacobian algebras of quivers with potentials, is a basic one. It was first solved for representation-finite cluster tilted algebras in [13], then for the cluster tilted algebras having a cyclically oriented quiver [9]. The latter class includes the representation-finite cluster tilted algebras. Also it was solved for Jacobian algebras arising from surfaces without punctures and in particular for cluster tilted algebras of type $\widetilde{\mathbb{A}}$ in [1]. We are not aware of other cases where the solution is known. We pose the following problem.

Problem 1. Given a system of minimal relations on a Jacobian algebra, which conditions are necessary on this system in order for the converse of Proposition 1.2.2 be valid?

1.3. Induced decompositions of the potential: the cyclically oriented case. Here we prove this converse in the two particular cases where systems of minimal relations are known. We start with algebras having cyclically oriented quivers. We recall from [9] that a quiver is called cyclically oriented if each chordless cycle is an oriented cycle. Here is a summary of the combinatorial properties of $\widetilde{Q}$ that follow from the fact that it is cyclically oriented (see [9, Proposition 1.1, Proposition 3.5]). 
(a) Let $a \in \widetilde{Q}_{1}$ lie in an oriented cycle. Then the sum of all the paths antiparallel to $a$ is a minimal relation.

(b) Any minimal relation is proportional to one as above.

(c) Let $a \in \widetilde{Q}_{1}$ lie in an oriented cycle. Then $a$ has no parallel arrow and two distinct paths antiparallel to $a$ have no common vertex but their source and target.

Here, two oriented paths, say from $x$ to $y$ and from $x^{\prime}$ to $y^{\prime}$, respectively, are called parallel whenever $x=x^{\prime}$ and $y=y^{\prime}$, and they are called antiparallel whenever $x=y^{\prime}$ and $y=x^{\prime}$.

Proposition 1.3.1. Let $\widetilde{C}$ be a cluster tilted algebra with a cyclically oriented quiver. Assume $E=E^{\prime} \oplus E^{\prime \prime}$ is a nontrivial direct sum decomposition of $E$ as $C-C$-bimodule. Then there exists a nontrivial direct sum decomposition $W=W^{\prime}+W^{\prime \prime}$ of the Keller potential such that $E^{\prime}, E^{\prime \prime}$ are respectively the partial relation bimodules corresponding to $W^{\prime}, W^{\prime \prime}$.

Proof. The direct sum decomposition of $C-C$-bimodules $E=E^{\prime} \oplus E^{\prime \prime}$ induces a decomposition top $E=\operatorname{top} E^{\prime} \oplus$ top $E^{\prime \prime}$. Let $\Sigma$ be the set of couples $(x, y)$ of vertices such that $\operatorname{Ext}_{C}^{2}\left(S_{x}, S_{y}\right) \neq 0$. Recall that $\operatorname{dim}_{\mathbb{k}} \operatorname{Ext}_{C}^{2}\left(S_{x}, S_{y}\right) \leqslant$ 1 for any couple $(x, y)$. Hence there exists a nontrivial partition $\Sigma=\Sigma^{\prime} \cup \Sigma^{\prime \prime}$ such that $e_{y} \operatorname{top}\left(E^{\prime}\right) e_{x}=e_{y} \operatorname{top}(E) x_{x}$ if $(x, y) \in \Sigma^{\prime}$ and $e_{y} \operatorname{top}\left(E^{\prime \prime}\right) e_{x}=$ $e_{y} \operatorname{top}(E) x_{x}$ if $(x, y) \in \Sigma^{\prime \prime}$. Since $\widetilde{Q}$ is cyclically oriented, if $(x, y) \in \Sigma$, then the arrow $y \rightarrow x$ in $Q_{\widetilde{C}}$ corresponding to the one-dimensional vector space $\operatorname{Ext}_{C}^{2}\left(S_{x}, S_{y}\right)$ is the unique path from $x$ to $y$ in $\widetilde{Q}$ (see [9]). In particular $e_{y} \cdot \operatorname{rad}(E) \cdot e_{x}=0$. Hence $e_{y} E e_{x}=e_{y} E^{\prime} e_{x}$ or $e_{y} E e_{x}=e_{y} E^{\prime \prime} e_{x}$ according to whether $(x, y) \in \Sigma^{\prime}$ or $(x, y) \in \Sigma^{\prime \prime}$.

For every couple $(x, y) \in \Sigma$, let $\alpha_{(x, y)}: y \rightarrow x$ be the corresponding arrow in $\widetilde{Q}$, let $r_{(x, y)} \in e_{x} \mathbb{k} Q e_{y}$ be a corresponding generator of $I$, and let $\xi_{(x, y)} \in \operatorname{Ext}_{C}^{2}\left(I_{x}, P_{y}\right)$ be a corresponding element in $\operatorname{Ext}_{C}^{2}(D C, C)$. Therefore we have

(i) $W=\sum_{(x, y) \in \Sigma} \alpha_{(x, y)} r_{(x, y)}$,

(ii) $E^{\prime}$ is generated by $\left\{\xi_{(x, y)} \mid(x, y) \in \Sigma^{\prime}\right\}$, and

(iii) $E^{\prime \prime}$ is generated by $\left\{\xi_{(x, y)} \mid(x, y) \in \Sigma^{\prime \prime}\right\}$.

Let $W^{\prime}=\sum_{(x, y) \in \Sigma^{\prime}} \alpha_{(x, y)} r_{(x, y)}$ and $W^{\prime \prime}=\sum_{(x, y) \in \Sigma^{\prime \prime}} \alpha_{(x, y)} r_{(x, y)}$. Hence $W=$ $W^{\prime}+W^{\prime \prime}$. To prove that this is a direct sum decomposition of $W$ inducing the direct sum decomposition $E=E^{\prime} \oplus E^{\prime \prime}$, it suffices to prove that no arrow of $\widetilde{Q}$ appears simultaneously in a cycle of $W^{\prime}$ and in a cycle of $W^{\prime \prime}$.

By contradiction, assume there exists an arrow $a$ appearing simultaneously in a cycle of $W^{\prime}$ and in a cycle of $W^{\prime \prime}$. Because of the definition of $W^{\prime}$ and $W^{\prime \prime}$, the arrow $a$ is distinct from any $\alpha_{(x, y)}$, for $(x, y) \in \Sigma$. Therefore we have

$$
\begin{aligned}
\partial_{a} W & =\partial_{a} W^{\prime}+\partial_{a} W^{\prime \prime} \\
& =\sum_{(x, y) \in \Sigma^{\prime}} \varphi_{(x, y)} \alpha_{(x, y)} \psi_{(x, y)}+\sum_{(x, y) \in \Sigma^{\prime \prime}} \varphi_{(x, y)} \alpha_{(x, y)} \psi_{(x, y)}
\end{aligned}
$$

where, in the second row, $\varphi_{(x, y)}$ and $\psi_{(x, y)}$ denote elements in $\mathbb{k} Q$. Note that each one of the two terms of this row is nonzero in $\mathbb{k}_{k} \widetilde{Q}$ because $\Sigma^{\prime}$ and $\Sigma^{\prime \prime}$ 
are nonempty. Since $\partial_{a} W \in \widetilde{I}$, the expression (11) yields that

$$
\sum_{(x, y) \in \Sigma^{\prime}} \varphi_{(x, y)} \alpha_{(x, y)} \psi_{(x, y)}+\widetilde{I}=\sum_{(x, y) \in \Sigma^{\prime \prime}} \varphi_{(x, y)} \alpha_{(x, y)} \psi_{(x, y)}+\widetilde{I}
$$

where the left-hand side lies in $E^{\prime}$ and the right-hand side lies in $E^{\prime \prime}$. Since $E^{\prime} \cap E^{\prime \prime}=0$ it follows that both terms

$$
\begin{aligned}
& \sum_{(x, y) \in \Sigma^{\prime}} \varphi_{(x, y)} \alpha_{(x, y)} \psi_{(x, y)} \quad \text { and } \\
& \sum_{(x, y) \in \Sigma^{\prime \prime}} \varphi_{(x, y)} \alpha_{(x, y)} \psi_{(x, y)}
\end{aligned}
$$

are nonzero and lie in $\widetilde{I}$. Considering (c) above, both are nontrivial linear combinations of partial derivatives of $W$ with respect to arrows parallel to a. This contradicts (c). Thus the decomposition $W=W^{\prime} \oplus W^{\prime \prime}$ is direct.

Moreover, in the present situation, the direct sum decompositions of the relation bimodule assume particularly nice forms.

Corollary 1.3.2. Let $\widetilde{C}$ be a cluster tilted algebra with cyclically oriented quiver. Assume $E=E^{\prime} \oplus E^{\prime \prime}$ is a direct sum decomposition. Then there exist direct sum decompositions $C_{C}=P^{\prime} \oplus P^{\prime \prime}$ and $D(C)_{C}=I^{\prime} \oplus I^{\prime \prime}$ such that $E^{\prime}=\operatorname{Ext}_{C}^{2}\left(I^{\prime}, P^{\prime}\right)$ and $E^{\prime \prime}=\operatorname{Ext}_{C}^{2}\left(I^{\prime \prime}, P^{\prime \prime}\right)$.

Proof. As explained in the proof of Proposition 1.3.1, given vertices $x, y$, the vector space $\operatorname{Ext}_{C}^{2}\left(D\left(C e_{x}\right), e_{y} C\right)$ has dimension 0 or 1 . The claimed decompositions of $D C$ and $C$ follow from this property.

Note that the corollary implies that $\operatorname{Ext}_{C}^{2}\left(I^{\prime \prime}, P^{\prime}\right)=\operatorname{Ext}_{C}^{2}\left(I^{\prime}, P^{\prime \prime}\right)=0$.

Example 1.3.3. Let $C$ be the tilted algebra given by the quiver

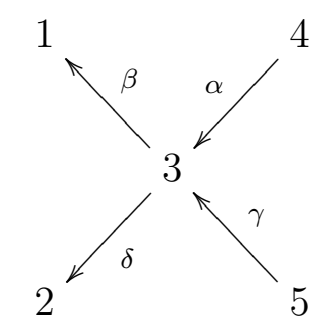

bound by $\alpha \beta=0, \gamma \delta=0$. It is easily verified that $E=\operatorname{Ext}_{C}^{2}(D C, C)=$ $\operatorname{Ext}_{C}^{2}\left(I_{4}, P_{1}\right) \oplus \operatorname{Ext}_{C}^{2}\left(I_{5}, P_{2}\right)$. Moreover $\widetilde{C}$ is given by the quiver

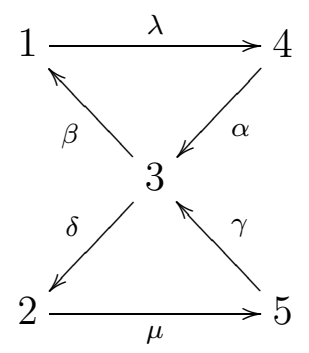

with potential $W=\alpha \beta \lambda+\gamma \delta \mu$. As seen in example (1.b) of 1.1, this is a direct sum decomposition of the potential $W$. It is easily seen that it 
corresponds to the direct sum decomposition $E=E^{\prime} \oplus E^{\prime \prime}$ with the summand $\alpha \beta \lambda$ corresponding to $E^{\prime}=\operatorname{Ext}_{C}^{2}\left(I_{4}, P_{1}\right)$ and $\delta \gamma \mu$ corresponding to $E^{\prime \prime}=\operatorname{Ext}_{C}^{2}\left(I_{5}, P_{2}\right)$.

1.4. Induced decompositions of the potential: the $\widetilde{\mathbb{A}}$ case. Another case where the Keller potential is known to induce a system of minimal relations is the case of cluster tilted algebras of type $\widetilde{\mathbb{A}}$ (see [1]). Therefore, in this case also we can deduce a decomposition of the Keller potential starting from a decomposition of the relation bimodule. The proof is different from that of the cyclically oriented case. It relies on the fact that the cluster tilted algebra $\widetilde{C}=\mathbb{k}_{\mathbb{Q}} \widetilde{Q} \widetilde{I}$ is gentle and on the following specific combinatorial properties of $\widetilde{Q}$.

Lemma 1.4.1. Let $i, j$ be vertices such that there exists an arrow $\alpha: i \rightarrow j$ in $\widetilde{Q} \backslash Q$ and such that $e_{i} \operatorname{rad}(E) e_{j} \neq 0$. Consider a path $u \beta v$ from $i$ to $j$ such that $u, v$ lie in $Q$ and are not both trivial, such that $\beta: i^{\prime} \rightarrow j^{\prime}$ is an arrow in $\widetilde{Q} \backslash Q$ and such that the class of $u \beta v$ in $e_{i} \operatorname{rad}(E) e_{j}$ is nonzero. Then

(1) no arrow is parallel to $\beta$ (or $\alpha$ ),

(2) $\alpha$ and $u \beta v$ are the only paths in $\widetilde{Q}$ not lying in $\widetilde{I}$, in particular $e_{i} E e_{j}$ is generated by $\alpha+\widetilde{I}$ and $u \beta v+\widetilde{I}$ and $e_{i} \operatorname{rad}(E) e_{j}$ is generated by $u \beta v+\widetilde{I}$, and

(3) $e_{i^{\prime}} \operatorname{rad}(E) e_{j^{\prime}}=0$.

Proof. (1) Should $\alpha$ have a parallel arrow $\alpha^{\prime}$, that arrow would lie in $\widetilde{Q}_{1} \backslash Q_{1}$. Since $(\widetilde{Q}, \widetilde{I})$ is a gentle bound quiver, the path $u \beta v$ would start with $\alpha$ or $\alpha^{\prime}$ and end with $\alpha$ and $\alpha^{\prime}$. The path $u \beta v$ would therefore contain two arrows from $\widetilde{Q}_{1} \backslash Q_{1}$ instead of only one, namely $\beta$. This proves that no arrow is parallel to $\alpha$.

By contradiction, assume that $\beta$ has a parallel arrow $\beta^{\prime}$. Then $\beta^{\prime}$ lies in $\widetilde{Q}_{1} \backslash Q_{1}$. Moreover $(\widetilde{Q}, \widetilde{I})$ contains the following bound quivers
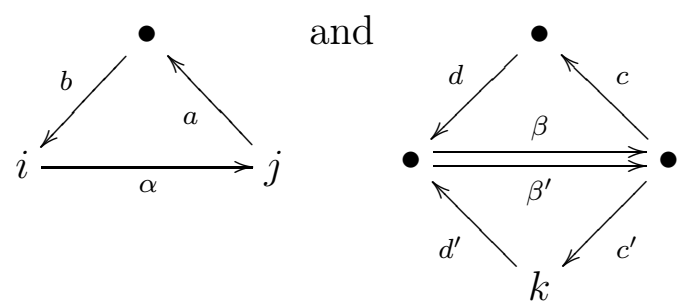

with relations all paths of length 2 in any triangle. Moreover, there exist paths $u^{\prime}$ and $v^{\prime}$ in $Q$ with sources $i$ and $k$, respectively, and with targets $k$ and $j$, respectively such that $u=u^{\prime} d^{\prime}$ and $v=c^{\prime} v^{\prime}$, and hence $u \beta v=u^{\prime} d^{\prime} \beta c^{\prime} v^{\prime}$. As a consequence, $\widetilde{C}$ contains the following two full subcategories that are hereditary of type $\widetilde{\mathbb{A}}$

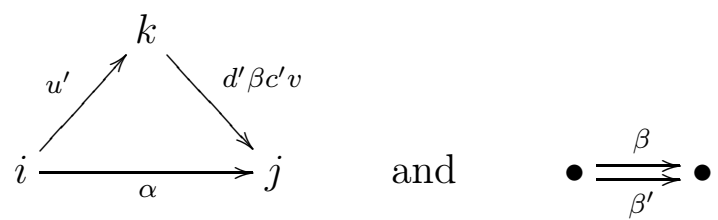


Note that these subcategories are indeed full because $(\widetilde{Q}, \widetilde{I})$ is a gentle bound quiver. The existence of these two subcategories is a contradiction to the characterisation of cluster tilted algebras of type $\widetilde{\mathbb{A}}$, see [1].

(2) This follows from the fact that $(\widetilde{Q}, \widetilde{I})$ is a gentle bound quiver.

(3) There only remains to prove that $e_{i^{\prime}} \operatorname{rad}(E) e_{j^{\prime}}=0$. If this was not the case, there would exist a path $w$ parallel to $\beta$, not lying in $Q$, and such that $w \notin \widetilde{I}$. According to $(2)$, the paths $\beta$ and $w$ would be the only paths in $\widetilde{Q}$ from $i^{\prime}$ to $j^{\prime}$. Hence $\widetilde{C}$ would have the following two full subcategories $i^{\prime} \underset{\beta}{\stackrel{w}{\rightleftarrows}} j^{\prime}$ and $i \underset{\alpha}{\stackrel{u \beta v}{\rightleftarrows}} j$. These are hereditary categories of type $\widetilde{\mathbb{A}}$. This would again contradict the classification of cluster tilted algebras of type $\widetilde{\mathbb{A}}$, see [1]. Thus $e_{i^{\prime}} \operatorname{rad}(E) e_{j^{\prime}}=0$.

Here is the construction of direct sum decomposition of the potential $W$ starting from direct sum decompositions of $E$ in the case of cluster tilted algebras of type $\widetilde{\mathbb{A}}$.

Proposition 1.4.2. Let $\widetilde{C}$ be a cluster tilted algebra of type $\widetilde{\mathbb{A}}$. Assume $E=E^{\prime} \oplus E^{\prime \prime}$ is a direct sum decomposition of $E$ as $C-C$-bimodule. Then there exists a direct sum decomposition $W=W^{\prime} \oplus W^{\prime \prime}$ of the Keller potential such that $E^{\prime}, E^{\prime \prime}$ are respectively the partial relation bimodules corresponding to $W^{\prime}, W^{\prime \prime}$.

Proof. Let $\Sigma$ be the set of couples of vertices $(x, y)$ such that $e_{x} \operatorname{top}(E) e_{y} \neq 0$. Note that $e_{x} \operatorname{top}(E) e_{y}$ has dimension at most 2 for any couple of vertices $(x, y)$ because $(\widetilde{Q}, \widetilde{I})$ is a gentle bound quiver. According to the preceding lemma, the set $\Sigma$ admits the partition $\Sigma=\Sigma_{1} \cup \Sigma_{2} \cup \Sigma_{3}$ where

- $\Sigma_{1}$ is the set of couples $(x, y)$ such that $e_{x} \operatorname{rad}(E) e_{y}=0$ and $e_{x} \operatorname{top}(E) e_{y}$ has dimension 1 ,

- $\Sigma_{2}$ is the set of couples $(x, y)$ such that $e_{x} \operatorname{rad}(E) e_{y} \neq 0$,

- $\Sigma_{3}$ is the set of couples $(x, y)$ such that $e_{x} \operatorname{rad}(E) e_{y}=0$ and $e_{x} \operatorname{top}(E) e_{y}$ has dimension 2 .

In what follows we make a detailed study of these sets. Note that if $(i, j) \in$ $\Sigma_{1}$ then $\operatorname{dim}\left(e_{i} E e_{j}\right)=1$. Therefore, $(i, j) \in \Sigma_{1}$ implies that

$$
\left\{\begin{array} { r l } 
{ e _ { i } E e _ { j } } & { = e _ { i } E ^ { \prime } e _ { j } } \\
{ 0 } & { = e _ { i } E ^ { \prime \prime } e _ { j } }
\end{array} \quad \text { or } \quad \left\{\begin{array}{rl}
e_{i} E e_{j} & =e_{i} E^{\prime \prime} e_{j} \\
0 & =e_{i} E^{\prime \prime} e_{j}
\end{array}\right.\right.
$$

Now let us study $\Sigma_{2}$. According to Lemma 1.4.1, and using the same notation, we have that $e_{i} E e_{j}$ is generated by $\alpha+\widetilde{I}$ and $u \beta v+\widetilde{I}$. Denote by $i^{\prime}$ and $j^{\prime}$ the source and target of $\beta$, respectively. Following Lemma 1.4.1, the couple $\left(i^{\prime}, j^{\prime}\right)$ lies in $\Sigma_{1}$. Without loss of generality we may assume that $e_{i^{\prime}} E e_{j^{\prime}}=e_{i^{\prime}} E^{\prime} e_{j^{\prime}}$ and $e_{i^{\prime}} E^{\prime \prime} e_{j^{\prime}}=0$ (see (2) $)$. Assume that $\alpha+\widetilde{I}$ does not lie in $E^{\prime} \cup E^{\prime \prime}$. Then there exists $\lambda \in \mathbb{k}^{\times}$such that

$$
\alpha+\widetilde{I}=(\lambda u \beta v+\widetilde{I})+((\alpha-\lambda u \beta v)+\widetilde{I})
$$


is the decomposition of $\alpha \bmod \widetilde{I}$ according to $E=E^{\prime} \oplus E^{\prime \prime}$. By construction of $\widetilde{C}$, the gentle bound quiver $(\widetilde{Q}, \widetilde{I})$ contains a bound quiver of the following shape

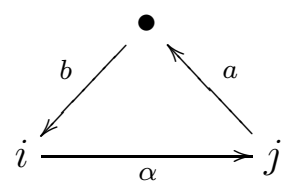

bound by $a b \in \widetilde{I}, b \alpha \in \widetilde{I}, \alpha a \in \widetilde{I}$. Therefore $u \beta v a \notin \widetilde{I}$ because the last arrow of $u \beta v$ is not $\alpha$. Hence $\alpha-\lambda u \beta v+\widetilde{I}$ is an element of the $C-C$-bimodule $E^{\prime \prime}$ satisfying

$$
(\alpha-\lambda u \beta+\widetilde{I}) \cdot(a+\widetilde{I})=\lambda u \beta v a+\widetilde{I} \in E^{\prime} \backslash\{0\} .
$$

Remember that $\beta+\widetilde{I} \in E^{\prime}$ by hypothesis. This contradicts the fact that the decomposition $E=E^{\prime} \oplus E^{\prime \prime}$ is direct. Thus, $(i, j) \in \Sigma_{2}$ implies that

$$
\alpha+\widetilde{I} \in E^{\prime} \cup E^{\prime \prime}
$$

where $\alpha: i \rightarrow j$ is the unique arrow of $\widetilde{Q}$ with source $i$ and target $j$. As a consequence, exactly one the following situations occurs when $(i, j) \in \Sigma_{2}$ :

(a) $e_{i} E^{\prime} e_{j}=\operatorname{Span}(\alpha+\widetilde{I}, u \beta v+\widetilde{I})$ and $e_{i} E^{\prime \prime} e_{j}=0$

(b) $e_{i} E^{\prime} e_{j}=\operatorname{Span}(\alpha+\widetilde{I})$ and $e_{i} E^{\prime \prime} e_{j}=\operatorname{Span}(u \beta v+\widetilde{I})$

(c) $e_{i} E^{\prime} e_{j}=\operatorname{Span}(u \beta v+\widetilde{I})$ and $e_{i} E^{\prime \prime} e_{j}=\operatorname{Span}(\alpha+\widetilde{I})$

(d) $e_{i} E^{\prime} e_{j}=0$ and $e_{i} E^{\prime \prime} e_{j}=\operatorname{Span}(\alpha+\widetilde{I}, u \beta v+\widetilde{I})$.

Let us finally consider a couple $(i, j) \in \Sigma_{3}$. Then $e_{i} \operatorname{rad}(E) e_{j}=0$ and $(\widetilde{Q}, \widetilde{I})$ contains a bound subquiver of the following shape

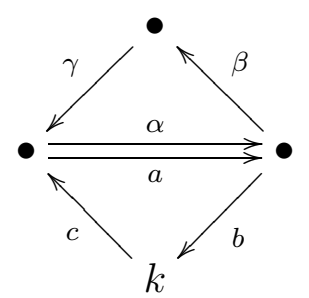

with relations $\alpha \beta, \beta \gamma, \gamma \alpha, a b, b c, c a \in \widetilde{I}$. Denote by $\bar{u}$ the class modulo $\widetilde{I}$ of a path $u$. Therefore $e_{i} E e_{j}=\operatorname{Span}(\bar{a}, \bar{\alpha})$. Let us prove that $e_{i} E^{\prime} e_{j}$ and $e_{i} E^{\prime \prime} e_{j}$ are one of the subspaces $0, \operatorname{Span}(\bar{a}), \operatorname{Span}(\bar{\alpha})$ or $\operatorname{Span}(\bar{a}, \bar{\alpha})$. If this is not the case, then there exists an invertible matrix $\left(\begin{array}{cc}t_{1} & t_{2} \\ t_{3} & t_{4}\end{array}\right)$ such that

$$
\begin{aligned}
& t_{1} \bar{a}+t_{2} \bar{\alpha} \in E^{\prime} \\
& t_{3} \bar{a}+t_{4} \bar{\alpha} \in E^{\prime \prime} \\
& \text { with } t_{1}, t_{2}, t_{3}, t_{4} \in \mathbb{R}^{\times} .
\end{aligned}
$$

This implies that

$$
\begin{aligned}
& 0 \neq t_{1} \overline{a \beta}=\left(t_{1} \bar{a}+t_{2} \bar{\alpha}\right) \bar{\beta} \in E^{\prime} \\
& 0 \neq t_{3} \overline{a \beta}=\left(t_{3} \bar{a}+t_{4} \bar{\alpha}\right) \bar{\beta} \in E^{\prime \prime} .
\end{aligned}
$$


This is absurd. Thus, if $(i, j) \in \Sigma_{3}$, then

$$
e_{i} E^{\prime} e_{j}, e_{i} E^{\prime \prime} e_{j} \in\{0, \operatorname{Span}(\bar{\alpha}), \operatorname{Span}(\bar{a}), \operatorname{Span}(\bar{\alpha}, \bar{a})\} .
$$

This study allows us to describe the claimed decomposition of $W$. Denote by $F$ the set of arrows in $\widetilde{Q}_{1} \backslash Q_{1}$. For every $\alpha \in F$ let $a_{\alpha} b_{\alpha} \in I$ be the associated monomial relation of length 2 in $(Q, I)$. Thus $W=\sum_{\alpha \in F} \alpha a_{\alpha} b_{\alpha}$. Remember that if $\alpha, \beta$ are distinct arrows lying in $F$, then $\alpha a_{\alpha} b_{\alpha}$ and $\beta a_{\beta} b_{\beta}$ have no common arrow because $(\widetilde{Q}, \widetilde{I})$ is a gentle bound quiver. It follows from (2), (3), (a), (b), (c), (d), and (4) that $\bar{\alpha} \in E^{\prime}$ or $\bar{\alpha} \in E^{\prime \prime}$, for every $\alpha \in F$. Denote by $F^{\prime}$ and $F^{\prime \prime}$ the subsets of $F$ consisting of the arrows $\alpha \in F$ such that $\bar{\alpha} \in E^{\prime}$ or $\bar{\alpha} \in E^{\prime \prime}$, respectively. This provides a partition $F=F^{\prime} \cup F^{\prime \prime}$. Moreover, the $C-C$-bimodules $E^{\prime}$ and $E^{\prime \prime}$ are generated by the classes modulo $\widetilde{I}$ of the arrows lying in $F^{\prime}$ and $F^{\prime \prime}$, respectively. Let $W^{\prime}=$ $\sum_{\alpha \in F^{\prime}} \alpha a_{\alpha} b_{\alpha}$ and $W^{\prime \prime}=\sum_{\alpha \in F^{\prime \prime}} \alpha a_{\alpha} b_{\alpha}$. The previous considerations show that $W=W^{\prime}+W^{\prime \prime}$ is a direct sum decomposition that fits the requirements of the proposition.

We now give an example showing that the analog of Corollary 1.3.2 does not hold true for cluster tilted algebras of type $\widetilde{\mathbb{A}}$. Assume that there exist decompositions $C=P^{\prime} \oplus P^{\prime \prime}$ and $D C=I^{\prime} \oplus I^{\prime \prime}$ such that $E^{\prime}=\operatorname{Ext}_{C}^{2}\left(I^{\prime}, P^{\prime}\right)$ and $E^{\prime \prime}=\operatorname{Ext}_{C}^{2}\left(I^{\prime \prime}, P^{\prime \prime}\right)$. Then, for any pair $(x, y)$ of points in $Q$, we have either $e_{x} E^{\prime} e_{y}=0$ or $e_{x} E^{\prime \prime} e_{y}=0$.

Example 1.4.3. Let $C$ be given by the quiver

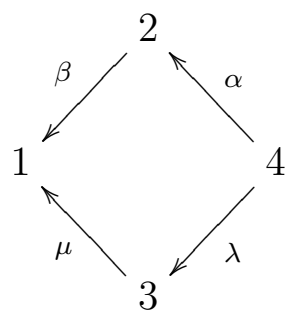

bound by all paths of length 2 . Then $\widetilde{C}$ is given by the quiver

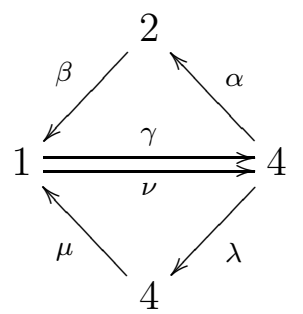

and the Keller potential is given by $W=\alpha \beta \gamma+\lambda \mu \nu$. The summands $\alpha \beta \gamma$ and $\lambda \mu \nu$ are independent, therefore the sum is direct and it induces a direct sum $E=E^{\prime} \oplus E^{\prime \prime}$ where $E^{\prime}=\operatorname{Span}(\gamma, \gamma \lambda, \mu \gamma, \mu \gamma \lambda)$ and $E^{\prime \prime}=$ $\operatorname{Span}(\nu, \nu \alpha, \beta \nu, \beta \nu \alpha)$. However we have $e_{1} E^{\prime} e_{4} \neq 0$ and $e_{1} E^{\prime \prime} e_{4} \neq 0$. This shows that Lemma 1.3.2 does not hold true in this case. 


\section{Partial Relation extension algebras}

2.1. The definition and examples. Let $C$ be a triangular algebra of global dimension at most 2 and $E^{\prime}$ be a direct summand of the $C-C$ bimodule $E=\operatorname{Ext}_{C}^{2}(D C, C)$. We recall that $\widetilde{C}=C \ltimes E$ is the relation extension of $C$. Then the trivial extension $B=C \ltimes E^{\prime}$ is called the partial relation extension of $C$ by $E^{\prime}$. In this subsection we prove a variant of transitivity for this construction. Let $E=E^{\prime} \oplus E^{\prime \prime}$ be a direct sum decomposition of the $C-C$-bimodule $E$ and $B=C \ltimes E^{\prime}$. Denote by $\pi: B \rightarrow C$ the canonical projection. Then $E^{\prime \prime}$ admits a $B-B$-bimodule structure by setting

$$
b_{1} x^{\prime \prime} b_{2}=\pi\left(b_{1}\right) x^{\prime \prime} \pi\left(b_{2}\right)
$$

for $b_{1}, b_{2} \in B$ and $x^{\prime \prime} \in E^{\prime \prime}$.

Lemma 2.1.1. With the preceding notation we have $\widetilde{C}=B \ltimes E^{\prime \prime}$.

Proof. We have an isomorphism of vector spaces:

$$
\varphi: \quad \begin{aligned}
C \ltimes E & \rightarrow\left(C \ltimes E^{\prime}\right) \ltimes E^{\prime \prime} \\
\left(c, e^{\prime}+e^{\prime \prime}\right) & \mapsto\left(\left(c, e^{\prime}\right), e^{\prime \prime}\right) .
\end{aligned}
$$

where $c \in C, e^{\prime} \in E^{\prime}$ and $e^{\prime \prime} \in E^{\prime \prime}$. It is necessary to check that

$$
\varphi\left(\left(c_{1}, e_{1}^{\prime}+e_{1}^{\prime \prime}\right)\left(c_{2}, e_{2}^{\prime}+e_{2}^{\prime \prime}\right)\right)=\varphi\left(c_{1}, e_{1}^{\prime}+e_{1}^{\prime \prime}\right) \varphi\left(c_{2}, e_{2}^{\prime}+e_{2}^{\prime \prime}\right) .
$$

Indeed

$$
\begin{aligned}
\varphi\left(c_{1}, e_{1}^{\prime}+e_{1}^{\prime \prime}\right) \varphi\left(c_{2}, e_{2}^{\prime}+e_{2}^{\prime \prime}\right) & =\left(\left(c_{1}, e_{1}^{\prime}\right), e_{1}^{\prime \prime}\right)\left(\left(c_{2}, e_{2}^{\prime}\right), e_{2}^{\prime \prime}\right) \\
& =\left(\left(c_{1}, e_{1}^{\prime}\right)\left(c_{2}, e_{2}^{\prime}\right),\left(c_{1}, e_{1}^{\prime}\right) e_{2}^{\prime \prime}+e_{1}^{\prime \prime}\left(c_{2}, e_{2}^{\prime}\right)\right) \\
& =\left(\left(c_{1} c_{2}, e_{1}^{\prime} c_{2}+c_{1} e_{2}^{\prime}\right), c_{1} e_{2}^{\prime \prime}+e_{1}^{\prime \prime} c_{2}\right) \\
& =\varphi\left(c_{1} c_{2}, e_{1}^{\prime} c_{2}+e_{1}^{\prime \prime} c_{2}+c_{1} e_{2}^{\prime}+c_{1} e_{2}^{\prime}\right) \\
& =\varphi\left(\left(c_{1}, e_{1}^{\prime}+e_{1}^{\prime \prime}\right)\left(c_{2}, e_{2}^{\prime}+e_{2}^{\prime \prime}\right)\right) .
\end{aligned}
$$

We pose the following problem on the meaning of $E^{\prime \prime}$ in terms of $C \ltimes E^{\prime}$.

Problem 2. Let $C$ be a triangular algebra of global dimension at most 2 and $E=E^{\prime} \oplus E^{\prime \prime}$ be a direct sum decomposition of the $C-C$-bimodule $E=\operatorname{Ext}_{C}^{2}(D C, C)$. What is the connection between $E^{\prime \prime}$ and the relation bimodule of the partial relation extension $C \ltimes E^{\prime}$ ?

Remark 2.1.2. We may define a poset of partial relation extensions. We say that $B_{1}=C \ltimes E_{1}$ is smaller than $B_{2}=C \ltimes E_{2}$ if $E_{1}$ is a direct summand of $E_{2}$. Notice that the obtained poset admits $\widetilde{C}$ as a unique maximal element and it admits $C$ as a unique minimal element. This poset is infinite in general. For instance, let $C$ be the algebra given by the following quiver

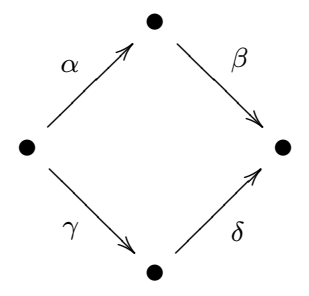


and relations $\alpha \beta, \gamma \delta$. Then, $\operatorname{dim}_{\mathbb{k}} E=2$. Let $(u, v)$ be a basis of $E$. For every point $[x: y]$ on the projective line $\mathbb{P}_{1}(\mathbb{k})$ denote by $B_{[x: y]}$ the partial relation extension of $C$ by the one-dimensional subbimodule of $E$ generated by $x u+$ $y v$. The resulting partial relation extensions are pairwise isomorphic. Then, the poset consists of the algebras $C, \widetilde{C}$ and $B_{[x: y]}$, for $[x: y] \in \mathbb{P}_{1}(\mathbb{k})$, and it has the following shape

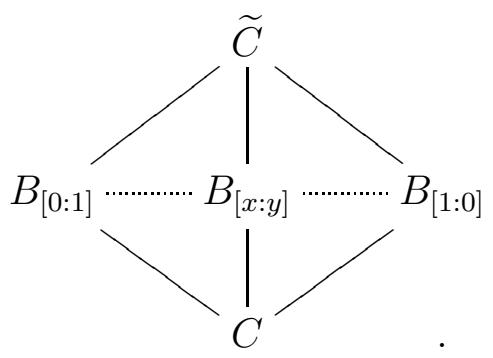

2.2. The bound quiver of a partial relation extension. Let $C=\mathbb{k} Q / I$ be a triangular algebra of global dimension at most two, let $\widetilde{C}=C \ltimes$ $\operatorname{Ext}_{C}^{2}(D C, C)$ be its relation extension, and assume that $E=\operatorname{Ext}_{C}^{2}(D C, C)$ has a $C-C$-bimodule direct sum decomposition $E=E^{\prime} \oplus E^{\prime \prime}$. Our objective is to describe a bound quiver presentation of the partial relation extension $B=C \ltimes E^{\prime}$ when this direct sum decomposition arises from a direct sum decomposition of the Keller potential associated with a minimal system of relations in $I$, see proposition 1.2.2.

Now, it follows from [2, (2.4)] that the new arrows generate the top of the $C-C$-bimodule $\operatorname{Ext}_{C}^{2}(D C, C)$. Assume that there exists a direct sum decomposition $W=W^{\prime} \oplus W^{\prime \prime}$ of the Keller potential in such a way that $E^{\prime}$ and $E^{\prime \prime}$ are the partial relation bimodules corresponding to $W^{\prime}$ and $W^{\prime \prime}$ respectively, see proposition 1.2 .2 . Then the set of new arrows can be partitioned into two sets $\left\{\alpha_{1}^{\prime}, \ldots, \alpha_{s}^{\prime}\right\}$ and $\left\{\alpha_{1}^{\prime \prime}, \ldots, \alpha_{t}^{\prime \prime}\right\}$ forming respectively the tops of $E^{\prime}$ and $E^{\prime \prime}$. We may now state.

Corollary 2.2.1. Let $C=\mathbb{k} Q / I$ be a triangular algebra of global dimension at most two, $\widetilde{C}$ its relation extension, $W$ the Keller potential associated with a minimal system of relations in $I$, and $\mathcal{J}$ the square of the ideal of $J(\widetilde{Q}, W)$ generated by the new arrows. If $E=E^{\prime} \oplus E^{\prime \prime}$ is a direct sum decomposition of $C-C$-bimodule arising from a direct sum decomposition of the Keller potential, $\alpha_{1}^{\prime \prime}, \ldots, \alpha_{t}^{\prime \prime}$ are the new arrows generating the top of $E^{\prime \prime}$ and $\mathcal{J}^{\prime}=\mathcal{J}+\sum_{i=1}^{t} \widetilde{C} \alpha_{i}^{\prime \prime} \widetilde{C}$. Then

$$
C \ltimes E^{\prime}=J(\widetilde{Q}, W) / \mathcal{J}^{\prime} .
$$

Proof. Let $B=C \ltimes E^{\prime}$. It follows from lemma 2.1.1 that $B \simeq \widetilde{C} / E^{\prime \prime}$. By definition, $E^{\prime \prime}$ is the subbimodule of $\operatorname{Ext}_{C}^{2}(D C, C)$ generated by the classes of the new arrows $\alpha_{1}^{\prime \prime}, \ldots, \alpha_{t}^{\prime \prime}$, see section [1.2. Hence the statement follows from the fact that $\widetilde{C} \simeq J(\widetilde{Q}, W) / \mathcal{J}$, see 1.2 ,

Thus, $B$ is given by the bound quiver obtained from that of $\widetilde{C}=\mathbb{k} \widetilde{Q} / \widetilde{I}$ by simply deleting the arrows $\alpha_{i}^{\prime \prime}$ from the ordinary quiver and by deleting any path involving such an arrow from any relation. Setting $W^{\prime}=\sum_{i=1}^{s} \rho_{i}^{\prime} \alpha_{i}^{\prime}$ and 
$W^{\prime \prime}=\sum_{i=1}^{t} \rho_{i}^{\prime \prime} \alpha_{i}^{\prime \prime}$ with $\alpha_{i}^{\prime}, \alpha_{j}^{\prime \prime}$ the new arrows and $\rho_{i}^{\prime}, \rho_{i}^{\prime \prime}$ the elements of the chosen minimal system of relations $R$ corresponding to $\alpha_{i}^{\prime}, \alpha_{j}^{\prime \prime}$ respectively, then the top of $E^{\prime}$ is generated by the $\alpha_{i}^{\prime}$ and the top of $E^{\prime \prime}$ is generated by the $\alpha_{j}^{\prime \prime}$, so we can state the following corollary.

Corollary 2.2.2. With the above notation, $B=C \ltimes E^{\prime}$ has a bound quiver as follows

(a) $\left(Q_{B}\right)_{o}=\underbrace{}_{Q_{o}}=\widetilde{Q}_{o}$,

(b) $\left(Q_{B}\right)_{1}=\widetilde{Q}_{1} \backslash\left\{\alpha_{1}^{\prime \prime}, \ldots, \alpha_{t}^{\prime \prime}\right\}=Q_{1} \cup\left\{\alpha_{1}^{\prime}, \ldots, \alpha_{s}^{\prime}\right\}$,

(c) the binding ideal $I_{B}$ is generated by the cyclic partial derivatives of $W^{\prime}$, the relations $\rho_{1}^{\prime \prime}, \ldots, \rho_{t}^{\prime \prime}$ and $\mathcal{J}$.

Example 2.2.3. Let $C$ be the tilted algebra given by the quiver

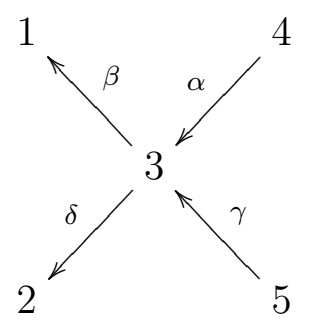

bound by $\alpha \beta=0, \gamma \delta=0$. Then $\widetilde{C}$ is the Jacobian algebra given by the quiver

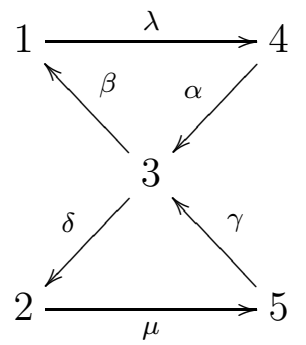

and the Keller potential $W=\alpha \beta \lambda+\gamma \delta \mu$. As seen in Section 1.1, $W^{\prime}=\alpha \beta \lambda$ and $W^{\prime \prime}=\gamma \delta \mu$ are independent so that $W=W^{\prime} \oplus W^{\prime \prime}$ is a direct sum decomposition. Setting $E^{\prime}=\operatorname{Ext}_{C}^{2}\left(I_{1}, P_{4}\right)$ and $E^{\prime \prime}=\operatorname{Ext}_{C}^{2}\left(I_{2}, P_{5}\right)$, then $E=$ $E^{\prime} \oplus E^{\prime \prime}$ is a direct sum decomposition of the bimodule $E=\operatorname{Ext}_{C}^{2}(D C, C)$ corresponding to the previous decomposition of the potential. The algebra $B=C \ltimes E^{\prime}$ is given by the quiver

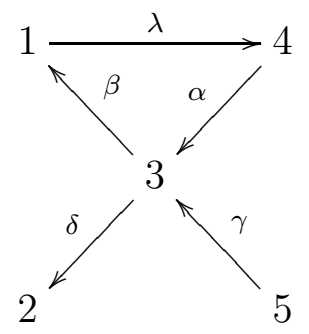

bound by $\alpha \beta=0, \gamma \delta=0, \lambda \alpha=0$ and $\beta \lambda=0$. 
2.3. The module category of a partial relation extension. In the present subsection, we assume that $C$ is tilted, so that its relation extension $\widetilde{C}$ is cluster tilted. Our objective is to give two descriptions of the module category of a partial relation extension, one as a quotient of a module category of a cluster tilted algebra, and the other as a quotient of another category which we now define. We mean by module a finitely generated right module. Given an algebra $B$ we denote by $\bmod B$ its module category.

We consider the following setting. Let $A$ be a hereditary algebra, $\mathcal{C}_{A}$ the corresponding cluster category and $T$ a cluster tilting object in $\mathcal{C}_{A}$. We denote by $\mathcal{D}^{b}(\bmod A)$ the bounded derived category of $\bmod A$ and by $\tau$ and $[-]$ respectively the Auslander-Reiten translation and the shift of $\mathcal{D}^{b}(\bmod A)$ respectively. Because of [12, Theorem 3.3$]$ we may assume that $T$ is actually a tilting module over $A$. We denote by $C=\operatorname{End}_{A}(T)$ the tilted algebra and by $\widetilde{C}=\operatorname{End}_{\mathcal{C}_{A}}(T)$. Then $\widetilde{C}$ is the relation extension of $C$.

We recall that it is shown in [2] that $E=\operatorname{Ext}_{C}^{2}(D C, C)$ is isomorphic to $\operatorname{Hom}_{\mathcal{D}^{b}(\bmod A)}\left(T, \tau^{-1} \circ T[1]\right)$ as a $C-C$-bimodule. Assume that $E=E^{\prime} \oplus E^{\prime \prime}$ is a $C-C$-bimodule direct sum decomposition. Observe that $E^{\prime}$ and $E^{\prime \prime}$ can be considered as subbimodules of $\operatorname{Hom}_{\mathcal{D}^{b}(\bmod A)}\left(T, \tau^{-1} \circ T[1]\right)$ and the latter may in turn be considered as contained in $\operatorname{End}_{\mathcal{C}_{A}}(T)=\widetilde{C}$, see [2].

Let $\mathcal{I}$ be the ideal of all morphisms in $\mathcal{C}_{A}$ generated by $E^{\prime \prime}$ that is, of all morphisms of $\mathcal{C}_{A}$ which factor through an element of $E^{\prime \prime}$ considered as a morphism from $T$ to $T$. We define $\mathcal{B}$ to be the additive quotient category $\mathcal{C}_{A}$ by $\mathcal{I}$, that is, $\mathcal{B}$ has the same objects as those of $\mathcal{C}_{A}$ and, if $X, Y$ are two such objects, then $\operatorname{Hom}_{\mathcal{B}}(X, Y)=\operatorname{Hom}_{\mathcal{C}_{A}}(X, Y) / \mathcal{I}(X, Y)$.

Proposition 2.3.1. With the above notation $\operatorname{End}_{\mathcal{B}}(T)$ is isomorphic to the partial relation extension $B=C \ltimes E^{\prime}$.

Proof. Because $\mathcal{B}=\mathcal{C}_{A} / \mathcal{I}$, we have $\operatorname{End}_{\mathcal{B}}(T)=\operatorname{End}_{\mathcal{C}_{A}}(T) / \mathcal{I}(T, T)$. However, as ideals of $\operatorname{End}_{\mathcal{C}_{A}}(T)$ we have $E^{\prime \prime}=\mathcal{I}(T, T)$. Hence $\operatorname{End}_{\mathcal{B}}(T) \simeq$ $\operatorname{End}_{\mathcal{C}_{A}}(T) / E^{\prime \prime} \simeq\left(C \ltimes\left(E^{\prime} \oplus E^{\prime \prime}\right)\right) / E^{\prime \prime} \simeq C \ltimes E^{\prime}$.

As a corollary, for every object $X$ in $\mathcal{B}$, the $\operatorname{End}_{\mathcal{B}}(T)$-module $\operatorname{Hom}_{\mathcal{B}}(T, X)$ is a $B$-module. Thus we have a functor $\operatorname{Hom}_{\mathcal{B}}(T,-): \mathcal{B} \rightarrow \bmod B$, which is full and dense. More precisely, we have the following lemma.

Lemma 2.3.2. We have a commutative diagram of full and dense functors

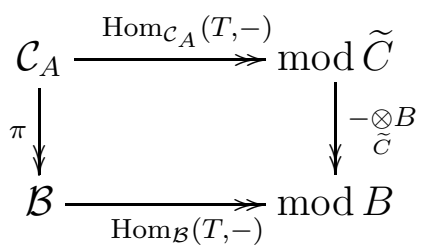

where $\pi: \mathcal{C}_{A} \rightarrow \mathcal{B}=\mathcal{C}_{A} / \mathcal{I}$ is the canonical projection.

Proof. The functor $-\underset{\widetilde{C}}{\otimes} B$ maps a $\widetilde{C}$-module $M$ to the $B$-module

$$
M \underset{\widetilde{C}}{\otimes} B=M \underset{\widetilde{C}}{\otimes} \widetilde{C} / E^{\prime \prime} \simeq M / M E^{\prime \prime} .
$$


Thus

$$
(-\underset{\widetilde{C}}{\otimes} B) \circ \operatorname{Hom}_{\mathcal{C}_{A}}(T,-)(X) \simeq \operatorname{Hom}_{\mathcal{C}_{A}}(T, X) / \operatorname{Hom}_{\mathcal{C}_{A}}(T, X) E^{\prime \prime} .
$$

On the other hand

$$
\begin{aligned}
\operatorname{Hom}_{\mathcal{B}}(T,-) \circ \pi(X) & =\operatorname{Hom}_{\mathcal{B}}(T, X) \\
& =\operatorname{Hom}_{\mathcal{C}_{A}}(T, X) / \mathcal{I}(T, X) .
\end{aligned}
$$

Now notice that $\mathcal{I}(T, X)$ is the image of the morphism $\operatorname{Hom}_{\mathcal{C}_{A}}(T, X) \otimes E^{\prime \prime} \rightarrow$ $\mathcal{I}(T, X)$ given by $u \otimes v \mapsto u \circ v$. Indeed, let $f \in \mathcal{I}(T, X)$, then $f=\sum_{i} u_{i} \circ e_{i} \circ v_{i}$ where $e_{i} \in E^{\prime \prime}, v_{i}: T \rightarrow E^{\prime \prime}$ and $u_{i}: E^{\prime \prime} \rightarrow X$. Because $\mathcal{I}(T, T)=E^{\prime \prime}$ is an ideal in $\operatorname{End}_{\mathcal{C}_{A}}(T)$, we have $e_{i} \circ v_{i} \in E^{\prime \prime}$. Therefore $f=\sum_{i} u_{i} \circ\left(e_{i} \circ v_{i}\right)$ belongs to the image of the given map. This shows that $\mathcal{I}(T, X)=\operatorname{Hom}_{\mathcal{C}_{A}}(T, X) E^{\prime \prime}$. The shown diagram is thus commutative.

Now, if $M$ is a $B$-module, then it admits a natural $\widetilde{C}$-module structure, and, with respect to this structure, $M \underset{\widetilde{C}}{\otimes} B \simeq M_{B}$. Thus the functor $-\underset{\widetilde{C}}{\otimes} B$ is full and dense. On the other hand, $\operatorname{Hom}_{\mathcal{C}_{A}}(T,-)$ is full and dense because of [14, Proposition 2.1]. Hence $\operatorname{Hom}_{\mathcal{B}}(T,-)$ is full and dense.

We now turn our attention to the kernel of the composed functor $(-\underset{\widetilde{C}}{\otimes}$ $B) \circ \operatorname{Hom}_{\mathcal{C}_{A}}(T,-): \mathcal{C}_{A} \rightarrow \bmod B$.

Lemma 2.3.3. The kernel of the composed functor $(-\underset{\widetilde{C}}{\otimes}) \circ \operatorname{Hom}_{\mathcal{C}_{A}}(T,-)$ is the ideal $\mathcal{K}$ of $\mathcal{C}_{A}$ consisting of all morphisms $f: X \stackrel{\widetilde{C}}{\rightarrow} Y$ such that the composition of $f$ with a minimal add $(T)$-approximation $u_{X}: T_{X} \rightarrow X$ can be written in the form $f \circ u_{X}=u_{Y} \circ$ e where $e \in E^{\prime \prime}$ and $u_{Y}: T_{Y} \rightarrow Y$ is a minimal add $(T)$-approximation.

Proof. Let $f: X \rightarrow Y$ be a morphism in $\mathcal{C}_{A}$. Using minimal add $(T)$-approximations of $X$ and $Y$ yields the following diagram in $\mathcal{C}_{A}$.

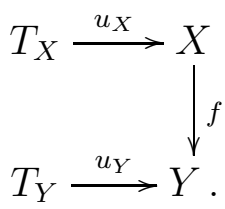

The image of $f$ in $\bmod B$ is equal to that of the mapping

$$
\operatorname{Hom}_{\mathcal{C}_{A}}(T, X) / \operatorname{Hom}_{\mathcal{C}_{A}}(T, X) E^{\prime \prime} \rightarrow \operatorname{Hom}_{\mathcal{C}_{A}}(T, Y) / \operatorname{Hom}_{\mathcal{C}_{A}}(T, Y) E^{\prime \prime}
$$

given by $\bar{u} \mapsto \overline{f \circ u}$, where the notation $\bar{g}$ stands for the residual class of a morphism $g$ in its respective quotient. If $\overline{f \circ u}$ vanishes for every $\bar{u}$ then it vanishes for $u=u_{X}$. Because $\overline{f \circ u_{X}}=0$, there exist $T_{0} \in \operatorname{add}(T), e_{0} \in E^{\prime \prime}$ and a morphism $g_{0}: T_{0} \rightarrow Y$ such that $f \circ u_{X}=g_{0} \circ e_{0}$. Because $u_{Y}$ is a minimal add $(T)$-approximation, $g_{0}$ factors through it and thus there exists a morphism $g^{\prime}: T_{0} \rightarrow T_{Y}$ such that $u_{Y} \circ g^{\prime}=g_{0}$. Setting $e=g^{\prime} \circ e_{0}$ we get 
that $e \in E^{\prime \prime}$ because the latter is an ideal and $u_{Y} \circ e=f \circ u_{X}$.

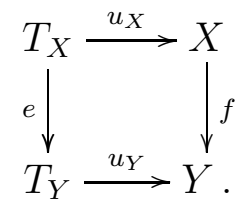

This proves that $f$ belongs to $\mathcal{K}$. Conversely if $f$ belongs to $\mathcal{K}$ then it is immediate that its image in $\bmod B$ is zero.

Theorem 2. The composed functor $(-\underset{\widetilde{C}}{\otimes} B) \circ \operatorname{Hom}_{\mathcal{C}_{A}}(T,-)$ induces an equivalence $\bmod B \simeq \mathcal{C}_{A} / \mathcal{K}$.

Proof. This follows immediately from lemmata 2.3.2 and 2.3.3.

Note that, taking $E^{\prime \prime}$ equal to 0 yields the main theorem of [14].

This theorem entails several consequences. Let $C$ be a tilted algebra. Recall that the cluster repetitive algebra is the locally finite dimensional algebra without identity

$$
\check{C}=\left(\begin{array}{ccccc}
\ddots & 0 & & & 0 \\
\ddots & C_{-1} & 0 & & \\
0 & E_{0} & C_{0} & 0 & \\
& 0 & E_{1} & C_{1} & \\
0 & & 0 & \ddots & \ddots
\end{array}\right)
$$

where the matrices have only finitely many nonzero entries, $C_{i}=C$ and $E_{i}=\operatorname{Ext}_{C}^{2}(D C, C)$ for all $i \in \mathbb{Z}$, all remaining entries are zero and the multiplication is induced from that of $C$, the $C-C$-bimodule structure of $\operatorname{Ext}_{C}^{2}(D C, C)$ and the zero map $\operatorname{Ext}_{C}^{2}(D C, C) \otimes \operatorname{Ext}_{C}^{2}(D C, C) \rightarrow 0$. The identity maps $C_{i} \rightarrow C_{i-1}$ and $E_{i} \rightarrow E_{i-1}$ induce an automorphism $\varphi$ of $\widetilde{C}$ and the orbit category $\check{C} /\langle\varphi\rangle$ inherits from $\check{C}$ a $\mathbb{k}$-algebra structure isomorphic to $\widetilde{C}=C \ltimes \operatorname{Ext}_{C}^{2}(D C, C)$. Thus the projection functor $G: \check{C} \rightarrow \widetilde{C}$ is a Galois covering with infinite cyclic group generated by $\varphi$. We denote by $G_{\lambda}: \bmod \check{C} \rightarrow \bmod \widetilde{C}$ the associated push-down functor (see [16]).

Now let $A$ be a hereditary algebra and $T$ be a tilting $A$-module such that $C=\operatorname{End}_{A}(T)$. Consider the automorphism $F=\tau^{-1} \circ[1]$ in $\mathcal{D}^{b}(\bmod A)$ and let $\pi^{\prime}: \mathcal{D}^{b}(\bmod A) \rightarrow \mathcal{C}_{A}$ denote the canonical projection onto the cluster category. We are now able to state the first corollary.

Corollary 2.3.4. With the above notation, there exists a commutative diagram of full and dense functors

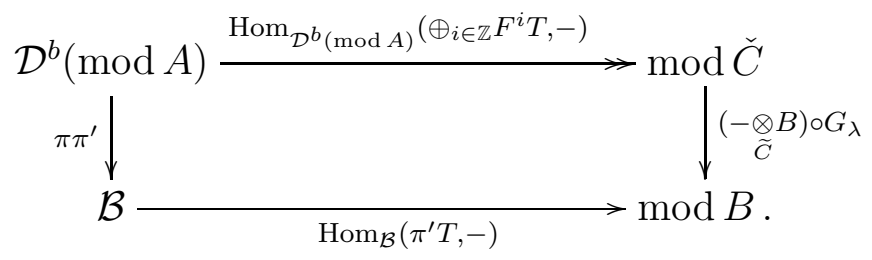


Proof. It is shown in [3, Theorem 9 of 2.3], that there is a commutative diagram of dense functors

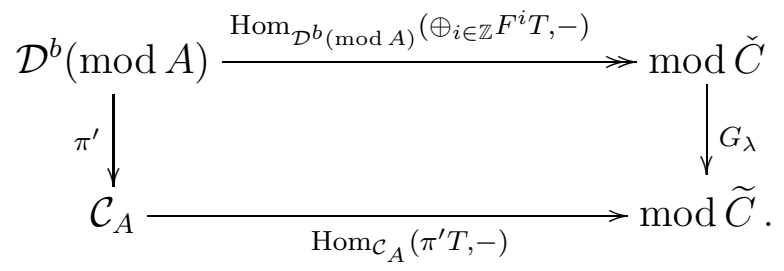

These functors are also full: $\pi^{\prime}$ is full by definition, $\operatorname{Hom}_{\mathcal{D}^{b}(\bmod A)}\left(\oplus_{i \in \mathbb{Z}} F^{i} T,-\right)$ is full because of [3, Proposition 7 of 2.1], and $\operatorname{Hom}_{\mathcal{C}_{A}}\left(\pi^{\prime} T,-\right)$ is full because of [14, Proposition 2.1]. The required commutative square follows upon composing this diagram with the one of lemma 2.3 .2 above.

As a consequence of this corollary, there is also a relation with the repetitive algebra $\widehat{C}$ of $C$, this is the algebra

$$
\widehat{C}=\left(\begin{array}{ccccc}
\ddots & 0 & & & 0 \\
\ddots & C_{-1} & 0 & & \\
0 & Q_{0} & C_{0} & 0 & \\
& 0 & Q_{1} & C_{1} & \\
0 & & 0 & \ddots & \ddots
\end{array}\right)
$$

where matrices have only finitely many nonzero entries, $C_{i}=C$ and $Q_{i}=$ $D C$ for all $i \in \mathbb{Z}$, all remaining entries are zero, addition is the usual addition of matrices and multiplication is induced from that of $C$, the $C-C$ bimodule structure of $D C$ and the zero maps $D C \otimes D C \rightarrow 0$. The Nakayama automorphism $\nu$ of $\widehat{C}$ is the one induced by the identity maps $C_{i} \rightarrow C_{i-1}$, $Q_{i} \rightarrow Q_{i-1}$. Then the quotient category $\widehat{C} /\langle\nu\rangle$ is isomorphic to the trivial extension $T(C)=C \ltimes D C$ of $C$ by its minimal injective cogenerator $D C$ (see [17]). There is a natural functor from $\bmod \widehat{C}$ to $\bmod \check{C}$ : Indeed, let $p: \bmod \widehat{C} \rightarrow \underline{\bmod } \widehat{C}$ denote the canonical projection, and define $\Phi: \bmod \widehat{C} \rightarrow \bmod \check{C}$ to be the composition

$$
\bmod \widehat{C} \stackrel{p}{\rightarrow} \underline{\bmod } \widehat{C} \stackrel{\underline{\operatorname{Hom}}_{\widehat{C}}}{\stackrel{(}{\left.\oplus_{i \in \mathbb{Z}} \tau^{i} \Omega^{-i} C,-\right)} \rightarrow} \bmod \check{C} .
$$

Corollary 2.3.5. With the above notation, there exists a commutative diagram of full and dense functors

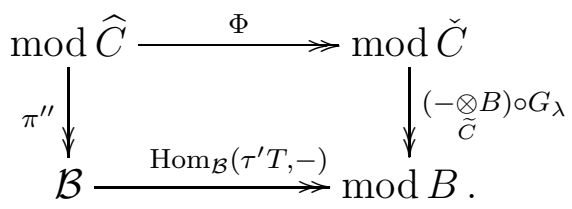

Proof. Let $\mathcal{C}_{C}$ be the orbit category of $\underline{\bmod } \widehat{C}$ under the action of the automorphism $F_{C}: \underline{\bmod } \widehat{C} \rightarrow \underline{\bmod } \widehat{C}$ defined by $F_{C}=\tau^{-1} \Omega^{-1}$ and the morphism space from $\left(F_{C}^{i} X\right)_{i \in \mathbb{Z}}$ to $\left(F_{C}^{i} Y\right)_{i \in \mathbb{Z}}$ is $\oplus_{i \in \mathbb{Z}} \underline{\operatorname{Hom}}_{\widehat{C}}\left(X, F_{C}^{i} Y\right)$. Also let $\widehat{\pi}$ be the composition of the two projection functors $p: \bmod \widehat{C} \rightarrow \underline{\bmod } \widehat{C}$ and 
$\underline{\widehat{\pi}}: \underline{\bmod } \widehat{C} \rightarrow \mathcal{C}_{C}$. Then, there is a commutative diagram of full and dense functors, see [3, Theorem 17 of 3.4$]$ :

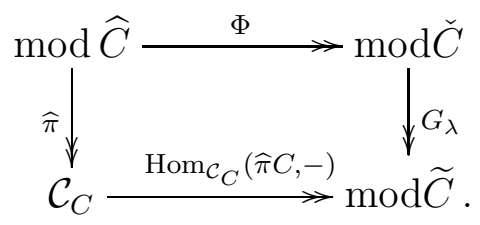

Moreover, it follows from [3, Lemma 15 of 3.2] that there is a commutative diagram of full and dense functors

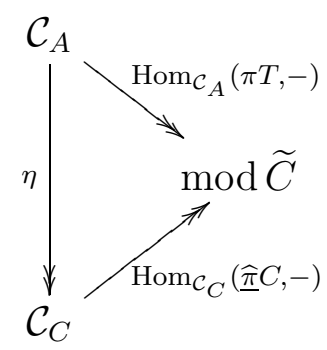

with $\eta$ an equivalence.

The required diagram follows upon composing these two diagrams with the one of lemma 2.3 .2 above. The functor $\pi^{\prime \prime}: \bmod \widehat{C} \rightarrow \mathcal{B}$ is equal to the composition $\pi \circ \eta^{-1} \circ \widehat{\pi}$.

Example 2.3.6.

(a) Let $C$ be the tilted algebra given by the quiver

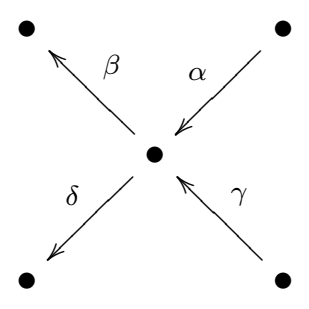

bound by $\alpha \beta=0, \gamma \delta=0$. Then its relation extension $\widetilde{C}$ is given by the quiver

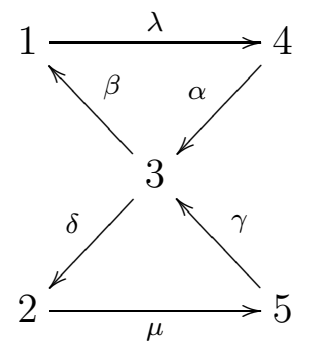

and the potential $W=\alpha \beta \lambda+\gamma \delta \mu$. As seen before in section 1.1, this is a direct sum decomposition $W=W_{1}+W_{2}$ with $W_{1}=\alpha \beta \lambda, W_{2}=\gamma \delta \mu$. Let $E^{\prime}$ be the direct summand of the $C-C$-bimodule $E=\operatorname{Ext}_{C}^{2}(D C, C)$ 
corresponding to $W_{1}$. Then $B=C \ltimes E^{\prime}$ is given by the quiver

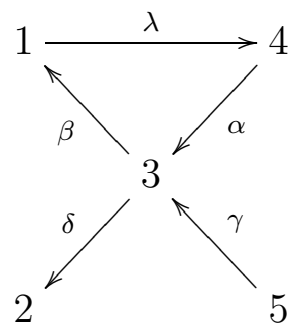

bound by $\alpha \beta=0, \beta \lambda=0, \gamma \delta=0, \lambda \alpha=0$. Its Auslander-Reiten quiver $\Gamma(\bmod B)$ is given by

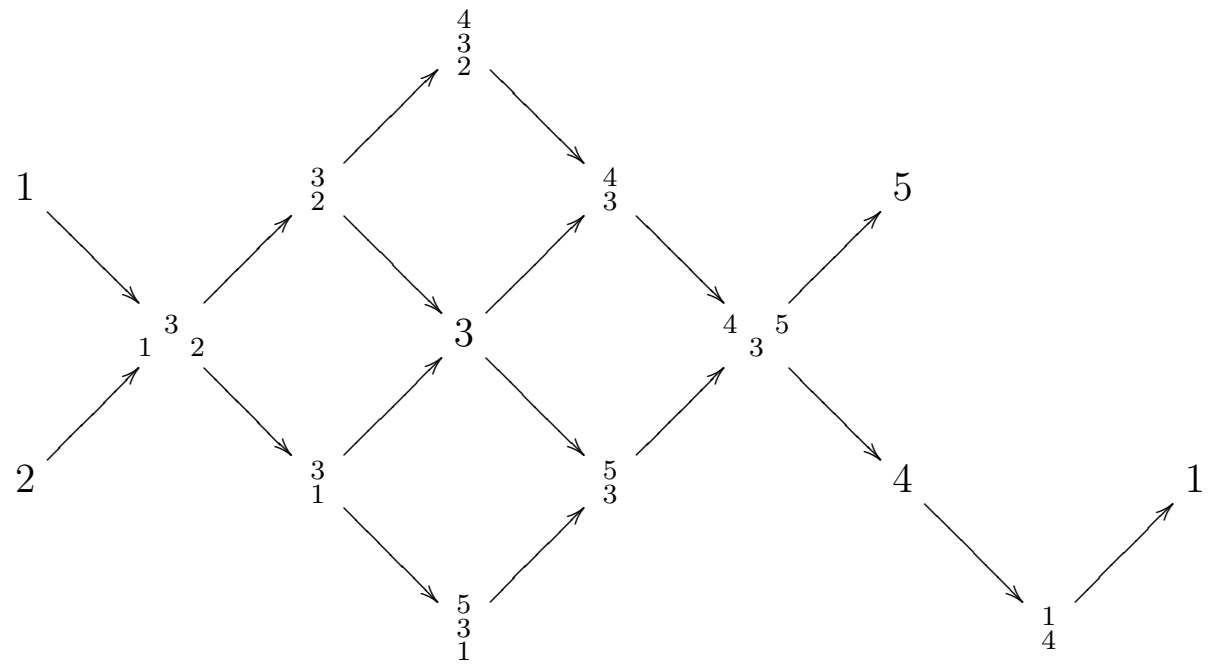

where the two copies of the simple $S_{1}=1$ are identified. The reader may compare this quiver with $\Gamma(\bmod \widetilde{C})$

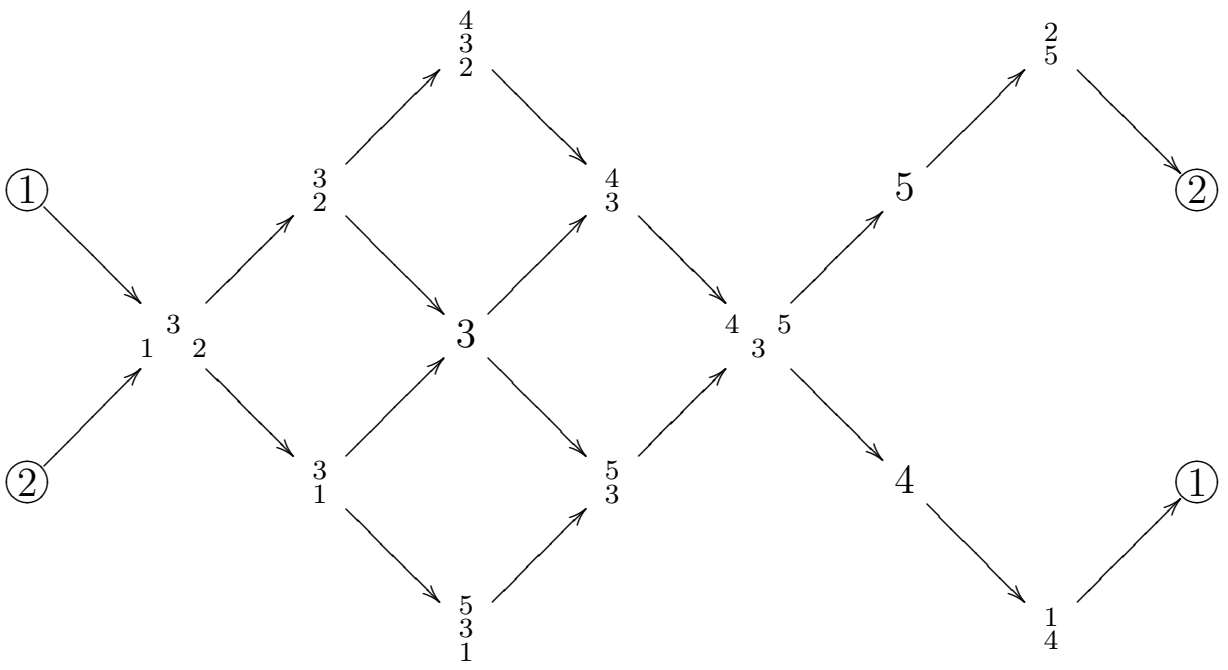

where the two encircled copies of $S_{1}=1$ are identified as are the two encircled copies of $S_{2}=2$. It is easily seen that $\Gamma(\bmod B)$ is obtained from $\Gamma(\bmod \widetilde{C})$ by deleting the $\widetilde{C}$-module $P_{2}={ }_{5}^{2}$. 
(b) Of course, one may have $\widetilde{C}$ representation-infinite but $B$ representationfinite. Let $C$ be given by the quiver

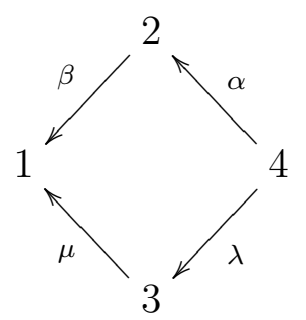

bound by $\alpha \beta=\underset{\sim}{0}, \lambda \mu=0$. Its relation extension $\widetilde{C}$ is the cluster tilted algebra of type $\widetilde{\mathbb{A}}$ given by the quiver

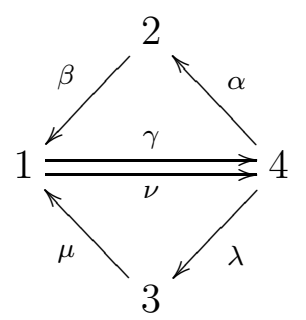

and the potential $W=\alpha \beta \gamma+\lambda \mu \nu$. This is a representation-infinite algebra. However, if we let $E^{\prime}$ be the direct summand of the $C-C$ bimodule corresponding to $W_{1}=\alpha \beta \gamma$, then $B=C \ltimes E^{\prime}$ is given by the quiver

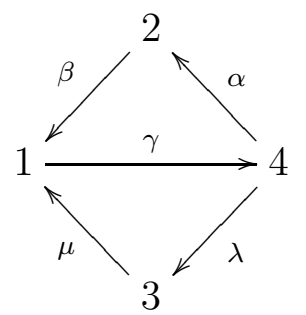

bound by $\alpha \beta=0, \beta \gamma=0, \gamma \alpha=0, \lambda \mu=0$. The algebra $B$ is representation-finite. Its Auslander-Reiten quiver is given by

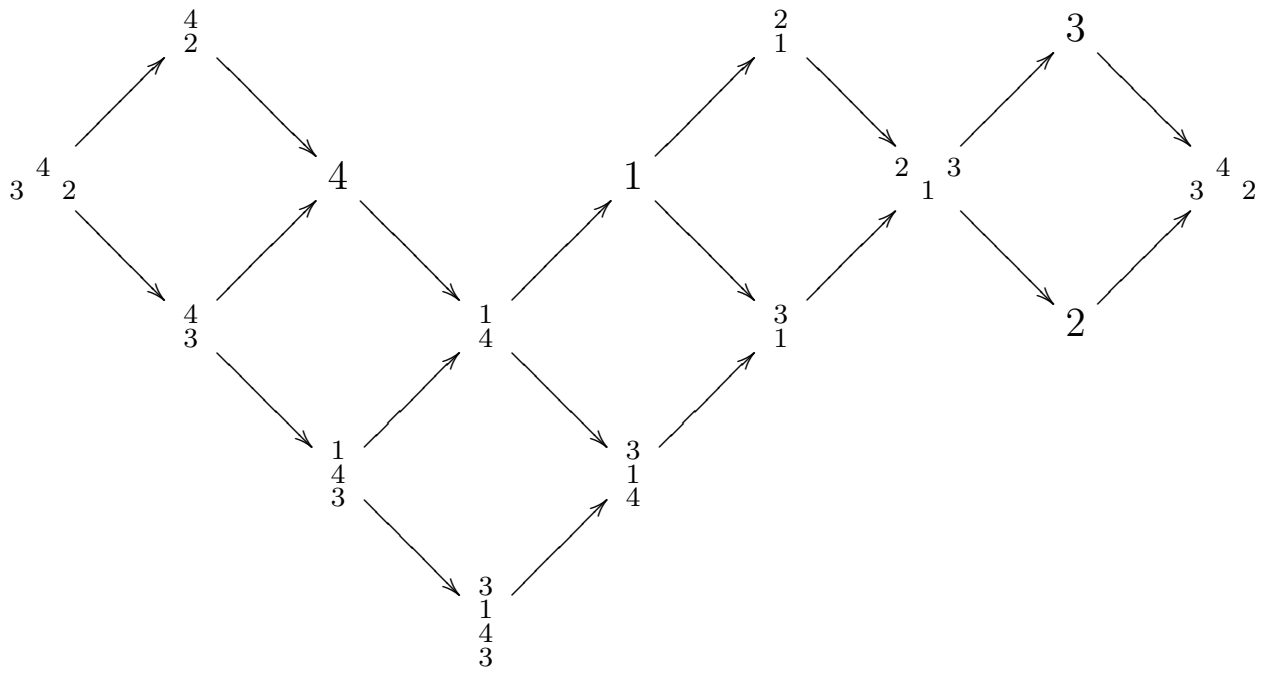


where the two copies of the module $P_{4}={ }_{3}{ }_{2}$ are identified.

\section{LOCAL SLICES}

3.1. Preliminary facts. The notion of local slice was defined in [4] for the study of cluster tilted algebras. We recall the definition.

Definition 3.1.1. Let $A$ be an algebra. A full subquiver $\Sigma$ of $\Gamma(\bmod A)$ is called a local slice if:

(1) It is a presection, that is, if $L \rightarrow M$ is an irreducible morphism between indecomposables in $\bmod A$, then

(a) $L \in \Sigma_{o}$ implies $M \in \Sigma_{o}$ or $\tau_{A} M \in \Sigma_{o}$,

(b) $M \in \Sigma_{o}$ implies $L \in \Sigma_{o}$ or $\tau_{A}^{-1} L \in \Sigma_{o}$.

(2) It is sectionally convex, that is, if $L=M_{0} \rightarrow M_{1} \rightarrow \cdots \rightarrow M_{n}=M$ is a sectional path in $\Gamma(\bmod A)$ such that $L, M \in \Sigma_{o}$, then $M_{i} \in \Sigma_{o}$ for all $i$.

(3) $\left|\Sigma_{o}\right|=\operatorname{rk}\left(K_{0}(A)\right)$.

Here $\left|\Sigma_{o}\right|$ denotes the number of points of $\Sigma$.

It is shown in [4] that, if $C$ is a tilted algebra, and $\Sigma$ is a complete slice in $\Gamma(\bmod C)$, then $\Sigma$ embeds fully as a local slice in $\Gamma(\bmod \widetilde{C})$, where $\widetilde{C}$ denotes, as usual, the relation extension of $C$, which is cluster tilted. However, local slices do not characterise cluster tilted algebras, and it was asked in 44 to identify the algebras which have local slices. Our objective in this section is to prove that, if $A$ is an algebra such that there exist surjective algebra morphisms $\widetilde{C} \rightarrow A \rightarrow C$, then $A$ admits a local slice in its Auslander-Reiten quiver. For this purpose, we need to recall the following well-known result of Auslander and Reiten, see [8, p. 187].

Proposition 3.1.2. Assume that there exists a surjective algebra homomorphism $A \rightarrow B$, and let $M$ be an indecomposable $B$-module. Then

(a) If $M$ is projective as an $A$-module, then $M$ is projective as a $B$-module.

If $M$ is not projective as an A-module, then $\tau_{B} M$ is a submodule of $\tau_{A} M$.

(b) If $M$ is injective as an $A$-module, then $M$ is injective as a B-module. If $M$ is not injective as an $A$-module then $\tau_{B}^{-1} M$ is a quotient of $\tau_{A}^{-1} M$.

3.2. Modules on slices. We start with the following lemma.

Lemma 3.2.1. Let $C$ be a tilted algebra, $M$ a module on a complete slice $\Sigma$ in $\Gamma(\bmod C)$ and $\widetilde{C}$ the relation extension of $C$. Then:

(a) If $M$ is projective as a $C$-module, then it is projective as a $\widetilde{C}$-module. If $M$ is not projective as a $C$-module then $\tau_{C} M \simeq \tau_{\widetilde{C}} M$.

(b) If $M$ is injective as a $C$-module, then it is injective as a $\widetilde{C}$-module. If $M$ is not injective as a $C$-module, then $\tau_{C}^{-1} M \simeq \tau_{\widetilde{C}}^{-1} M$.

Proof. We only prove (a) because the proof of (b) is dual. Assume first that $M=e C$ is projective, with $e$ a primitive idempotent of $C$. Let, as usual, $E=\operatorname{Ext}_{C}^{2}(D C, C)$. Because $\widetilde{C}=C \ltimes E$, it follows from [7, Corollary 1.4], 
that $M$ is projective as a $\widetilde{C}$-module if and only if $e E=0$. Now $e E=$ $e \operatorname{Ext}_{C}^{2}(D C, C)=\operatorname{Ext}_{C}^{2}(D C, e C) \simeq \operatorname{Ext}_{C}^{2}(D C, M)=0$ because $M$, lying on a complete slice in $\bmod C$, has injective dimension at most one.

Assume now that $M$ is not projective. It follows from [7, Theorem 2.1] that $\tau_{C} M \simeq \tau_{\widetilde{C}} M$ if and only if $M \underset{C}{\otimes} E=0$ and $\operatorname{Hom}_{C}\left(E, \tau_{C} M\right)=0$. We proceed to prove these two equalities.

Because $C$ is tilted and $\Sigma$ is a complete slice, the algebra $H=\operatorname{End}_{C}\left(\oplus_{U \in \Sigma_{o}} U\right)$ is hereditary and there exists a tilting $H$-module $T$ such that $C=\operatorname{End}_{H}(T)$. Because $M \in \Sigma_{o}$, there exists an injective $H$-module $I$ such that $M=$ $\operatorname{Hom}_{H}(T, I)$, see [6, (VIII.3.5) and (VIII.5.6)]. Denoting as before by [-] the shift functor in the bounded derived category $\mathcal{D}^{b}(\bmod H)$ and by $\tau$ its Auslander-Reiten translation, it follows from [2] that:

$$
\begin{aligned}
D\left(M \stackrel{C}{Q}_{E} E\right) & \simeq \operatorname{Hom}_{C}(M, D E) \\
& \simeq \operatorname{Hom}_{C}\left(\operatorname{Hom}_{H}(T, I), D \operatorname{Hom}_{\mathcal{D}^{b}(\bmod H)}\left(T, \tau^{-1} T[1]\right)\right) \\
& \simeq \operatorname{Hom}_{C}\left(\operatorname{Hom}_{H}(T, I), D \operatorname{Hom}_{\mathcal{D}^{b}(\bmod H)}(\tau T, T[1])\right) \\
& \simeq \operatorname{Hom}_{C}\left(\operatorname{Hom}_{H}(T, I), D \operatorname{Ext}_{\mathcal{D}^{b}(\bmod H)}(\tau T, T)\right) \\
& \simeq \operatorname{Hom}_{C}\left(\operatorname{Hom}_{H}(T, I), \operatorname{Hom}_{H}\left(T, \tau_{H}^{2} T\right)\right) \\
& \simeq \operatorname{Hom}_{H}\left(I, t\left(\tau_{H}^{2} T\right)\right)
\end{aligned}
$$

where $t\left(\tau_{H}^{2} T\right)=\operatorname{Hom}_{H}\left(T, \tau_{H}^{2} T\right){\underset{C}{Q}}_{T} T$ is the torsion submodule of $\tau_{H}^{2} T$ in the torsion pair $\left(\mathcal{T}\left(T_{H}\right), \mathcal{F}\left(T_{H}\right)\right)$ induced by $T$ in $\bmod H$, see [6, (VI.3.9)]. Now $\tau_{H}^{2} T$ is clearly not injective, therefore neither is its submodule $t\left(\tau_{H}^{2} T\right)$. Because $I$ is injective and $H$ is hereditary, we infer that $\operatorname{Hom}_{H}\left(T, t\left(\tau_{H}^{2} T\right)\right)=$ 0 . Therefore $M \underset{C}{\otimes} E=0$.

The proof that $\operatorname{Hom}_{C}\left(E, \tau_{C} M\right)=0$ is sensibly different. We first claim that every indecomposable summand of $E_{C}$ is a proper successor of the complete slice $\Sigma$. Indeed, the Auslander-Reiten formula yields

$$
E=\operatorname{Ext}_{C}^{2}(D C, C) \simeq \operatorname{Ext}_{C}^{1}\left(D C, \Omega^{-1} C\right) \simeq D \underline{\operatorname{Hom}}_{C}\left(\tau_{C}^{-1} \Omega^{-1} C, D C\right) .
$$

Now for any indecomposable summand $N$ of $\Omega^{-1} C$, there exists an indecomposable injective $C$-module $I_{0}$ such that $\operatorname{Hom}_{C}\left(I_{0}, N\right) \neq 0$. Because the slice $\Sigma$ is sincere in $\bmod C$, there exist $L \in \Sigma_{o}$ and a nonzero morphism $L \rightarrow I_{0}$. Thus we have a path $L \rightarrow I_{0} \rightarrow N \rightarrow \star \rightarrow \tau_{C}^{-1} N$ in $\bmod C$, so that $\tau_{C}^{-1} N$ is a proper successor of $\Sigma$ in $\bmod C$. This proves that any indecomposable summand of $\tau_{C}^{-1} \Omega^{-1} C$ is a proper successor of $\Sigma$ in $\bmod C$. On the other hand, no indecomposable projective $C$-module is a proper successor of $\Sigma$. Therefore

$$
\underline{\operatorname{Hom}}_{C}\left(\tau_{C}^{-1} \Omega^{-1} C, D C\right) \simeq \operatorname{Hom}_{C}\left(\tau_{C}^{-1} \Omega^{-1} C, D C\right)
$$

and so $E \simeq \operatorname{Hom}_{C}\left(\tau_{C}^{-1} \Omega^{-1} C, D C\right) \simeq \tau_{C}^{-1} \Omega^{-1} C$. This establishes our claim that every indecomposable summand of $E$ is a proper successor of $\Sigma$.

Now $\tau_{C} M$ is a proper predecessor of $\Sigma$. Therefore $\operatorname{Hom}_{C}\left(E, \tau_{C} M\right)=0$. This completes the proof. 
Proposition 3.2.2. Let $C$ be a tilted algebra, $M$ a module in a complete slice $\Sigma$ in $\Gamma(\bmod C), \widetilde{C}$ the relation extension algebra and $A$ an algebra such that there exist surjective algebra morphisms $\widetilde{C} \rightarrow A \rightarrow C$. Then:

(a) If $M$ is projective as a $C$-module, then it is projective as an $A$-module.

If $M$ is not projective as a $C$-module, then $\tau_{C} M \simeq \tau_{A} M$.

(b) If $M$ is injective as a $C$-module, then it is injective as an A-module. If $M$ is not injective as a $C$-module, then $\tau_{C}^{-1} M \simeq \tau_{A}^{-1} M$.

Proof. This follows from lemma 3.2.1 and proposition 3.1.2.

Corollary 3.2.3. Let $C$ be a tilted algebra, $\Sigma$ a complete slice in $\Gamma(\bmod C)$, $\widetilde{C}$ the relation extension of $C$ and $A$ an algebra such that there exist surjective algebra morphisms $\widetilde{C} \rightarrow A \rightarrow C$. Let $L \rightarrow M$ be an irreducible morphism between indecomposables in $\bmod A$. If either $L$ or $M$ lies in $\Sigma$, then the other is a $C$-module.

Proof. We may, by duality, assume that $L \in \Sigma_{o}$. Suppose first that $L$ is an injective $C$-module. Because of proposition 3.2.3, it is injective as an $A$-module. In particular, $\operatorname{soc}_{C} L=\operatorname{soc}_{A} L$ and so the canonical projection $L \rightarrow L / \operatorname{soc}_{C} L$ is a minimal left almost split morphism in $\bmod A$. Therefore $M$ is an indecomposable direct summand of $L / \operatorname{soc}_{C} L$ and in particular is a $C$-module.

Suppose that $L$ is not injective as a $C$-module. Because of proposition [3.2.3, we have $\tau_{C}^{-1} L \simeq \tau_{A}^{-1} L$. It then follows from [7, Theorem 2.1] that the almost split sequence $0 \rightarrow L \rightarrow X \rightarrow \tau_{C}^{-1} L \rightarrow 0$ in $\bmod C$ remains almost split in $\bmod A$. Therefore $M$ is an indecomposable direct summand of $X$, so it is a $C$-module. This completes the proof.

3.3. The existence of local slices. We are now able to prove the main result of this section.

Theorem 3. Let $C$ be a tilted algebra and $A$ be an algebra such that there exist surjective algebra morphisms $\widetilde{C} \rightarrow A \rightarrow C$, then any complete slice in $\Gamma(\bmod C)$ embeds as a local slice in $\Gamma(\bmod A)$. In particular, partial relation extensions admit local slices.

Proof. Because clearly $\left|\Sigma_{o}\right|=\operatorname{rk}\left(K_{0}(C)\right)=\operatorname{rk}\left(K_{0}(A)\right)$, it suffices to prove the first two properties in the definition of local slices.

We first show that $\Sigma$ is a presection in $\Gamma(\bmod A)$. Let $f: L \rightarrow M$ be an irreducible morphism between indecomposables in $\bmod A$. Assume $L \in \Sigma$. Because of corollary $3.2 .3, M$ is a $C$-module. Therefore $f$ remains an irreducible morphism in $\bmod C$. Because the complete slice $\Sigma$ is a presection in $\Gamma(\bmod C)$, we have $M \in \Sigma_{o}$ or $\tau_{C} M \in \Sigma_{o}$. In the latter case, the observation that $\tau_{C} M \simeq \tau_{A} M$ completes the proof.

One shows in exactly the same way that, if $M \in \Sigma_{o}$, then $L \in \Sigma_{o}$ or $\tau_{A}^{-1} L \in \Sigma_{o}$.

There remains to prove sectional convexity. Let

$$
M_{0} \stackrel{f_{1}}{\rightarrow} M_{1} \stackrel{f_{2}}{\rightarrow} M_{2} \rightarrow \cdots \stackrel{f_{t}}{\rightarrow} M_{t}
$$


be a sectional path in $\Gamma(\bmod A)$, with $M_{0}, M_{t} \in \Sigma$. We may assume without loss of generality that $M_{1} \notin \Sigma_{o}$. Because of corollary [3.2.3, $M_{1}$ is a $C$ module. Now, observe that the morphism $f_{t} \cdots f_{2}: M_{1} \rightarrow M_{t}$ is nonzero in $\bmod A$, because it is the composition of a sectional path. Therefore it is also nonzero in $\bmod C$. Because $f_{1}: M_{0} \rightarrow M_{1}$ is also nonzero in $\bmod C$, the convexity of $\Sigma$ in $\bmod C$ and the path $M_{0} \rightarrow M_{1} \rightarrow M_{t}$ yield $M_{1} \in \Sigma_{o}$, a contradiction which completes the proof.

In particular, our result applies to partial relation extensions.

Corollary 3.3.1. Let $C$ be a tilted algebra and $B$ a partial relation extension. Then any complete slice in $\Gamma(\bmod C)$ embeds as a local slice in $\Gamma(\bmod B)$.

The reader may notice that the example in [4] of a local slice is an example of a local slice in a partial relation extension. We give an example of an algebra which has a local slice but is not a partial relation extension.

Example 3.3.2. Let $A$ be given by the quiver

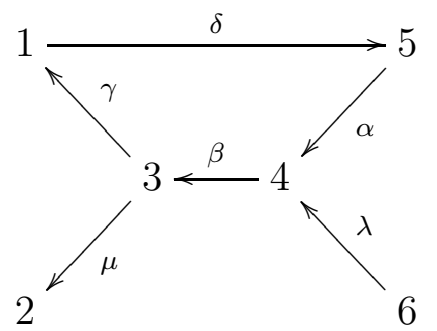

bound by $\lambda \beta \mu=0, \alpha \beta \gamma=0, \gamma \delta=0, \delta \alpha=0$. Then $\Gamma(\bmod A)$ is given by

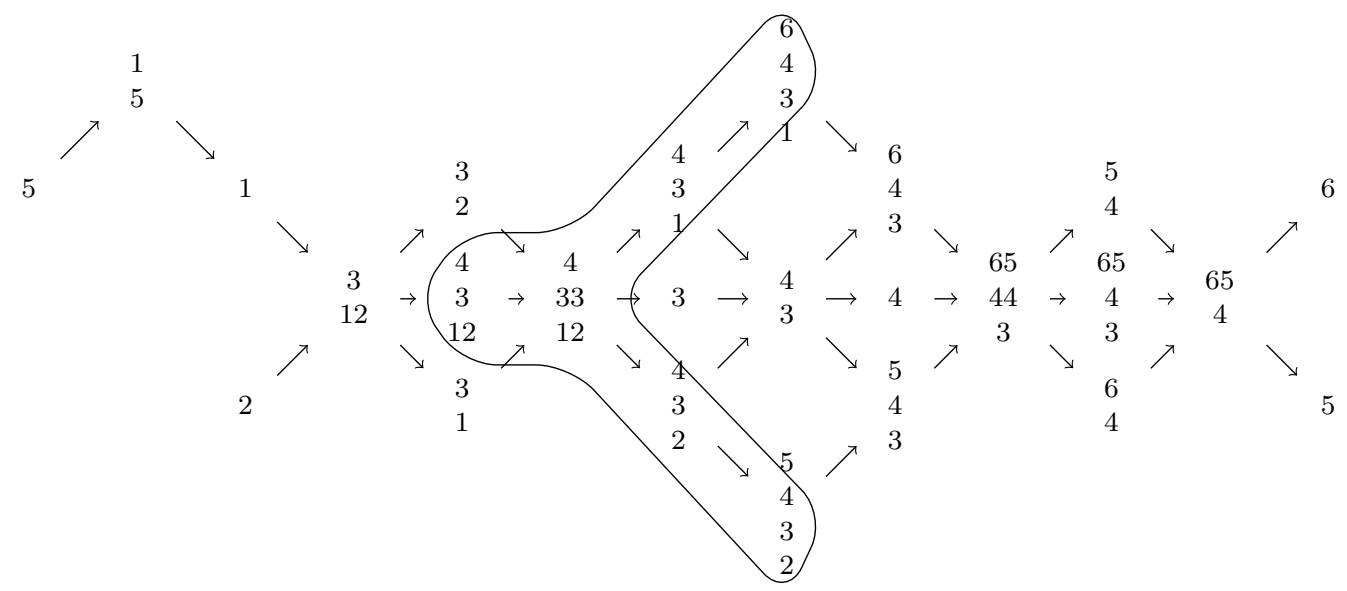

where the two copies of 5 are identified. We have illustrated a local slice which arises from the embedding of $\Gamma(\bmod C)$ in $\Gamma(\bmod A)$, where $C$ is the algebra obtained from $A$ by deleting the arrow $\delta$ (that is, $C=A /\langle\delta\rangle$ ). Notice that $C$ is a tilted algebra of type $\mathbb{E}_{6}$. Notice finally that $A$ is not a partial relation extension as shown by direct inspection: for instance, if $A$ were a partial relation extension of $C$, then the defining relations of $A$ involving the arrow $\gamma$ would be $\beta \gamma \delta=0$ and $\delta \alpha \beta=0$ (instead of $\gamma \delta=0$ and 
$\delta \alpha=0)$ because the Keller potential has a unique oriented cycle, namely, $\alpha \beta \gamma \delta$, containing $\alpha$ or $\gamma$.

\section{ACKNOWLEDGEMENTS}

Part of this work was done during visits of the fourth named author to his coauthors at Université de Sherbrooke. He thanks them for their warm hospitality during his stays.

The first named author gratefully acknowledges partial support from the NSERC of Canada, FRQNT of Québec and the Université de Sherbrooke.

The fourth named author acknowledges financial support from CRM (UMI CNRS 3457).

The fifth named author acknowledges financial support from the NSERC of Canada.

\section{REFERENCES}

[1] Ibrahim Assem, Thomas Brüstle, Gabrielle Charbonneau-Jodoin, and Pierre-Guy Plamondon. Gentle algebras arising from surface triangulations. Algebra Number Theory, 4(2):201-229, 2010.

[2] Ibrahim Assem, Thomas Brüstle, and Ralf Schiffler. Cluster-tilted algebras as trivial extensions. Bull. Lond. Math. Soc., 40(1):151-162, 2008.

[3] Ibrahim Assem, Thomas Brüstle, and Ralf Schiffler. On the Galois coverings of a cluster-tilted algebra. J. Pure Appl. Algebra, 213(7):14501463, 2009.

[4] Ibrahim Assem, Thomas Brüstle, and Ralf Schiffler. Cluster-tilted algebras and slices. J. Algebra, 319(8):3464-3479, 2008.

[5] Ibrahim Assem, Maria Andrea Gatica, Ralf Schiffler, and Rachel Taillefer. Hochschild cohomology of relation extension algebras. J. Pure Appl. Algebra, 220(7):2571-2499, 2016.

[6] Ibrahim Assem, Daniel Simson, and Andrzej Skowroński. Elements of the representation theory of associative algebras. Vol. 1, volume 65 of London Mathematical Society Student Texts. Cambridge University Press, Cambridge, 2006. Techniques of representation theory.

[7] Ibrahim Assem and Dan Zacharia. Full embeddings of almost split sequences over split-by-nilpotent extensions. Colloq. Math., 81(1):2131, 1999.

[8] Maurice Auslander, Idun Reiten, and Smalø Sverre O. Representation theory of Artin algebras, volume 36 of Cambridge Studies in Advanced Mathematics. Cambridge University Press, Cambridge, 1997. Corrected reprint of the 1995 original. 
[9] Michael Barot and Sonia Trepode. Cluster tilted algebras with a cyclically oriented quiver. Comm. Algebra, 41(10):3613-3628, 2013.

[10] Klaus Bongartz. Algebras and quadratic forms. J. London Math. Soc. (2), 28(3):461-469, 1983.

[11] Aslak Bakke Buan, Osamu Iyama, Idun Reiten, and David Smith. Mutation of cluster-tilting objects and potentials. Amer. J. Math., 133(4):835-887, 2011.

[12] Aslak Bakke Buan, Robert Marsh, Markus Reineke, Idun Reiten, and Gordana Todorov. Tilting theory and cluster combinatorics. Adv. Math., 204(2):572-618, 2006.

[13] Aslak Bakke Buan, Robert J. Marsh, and Idun Reiten. Cluster-tilted algebras of finite representation type. J. Algebra, 306(2):412-431, 2006.

[14] Aslak Bakke Buan, Robert J. Marsh, and Idun Reiten. Cluster-tilted algebras. Trans. Amer. Math. Soc., 359(1):323-332 (electronic), 2007.

[15] Philippe Caldero, Frédéric Chapoton, and Ralf Schiffler. Quivers with relations arising from clusters $\left(\mathbb{A}_{n}\right.$ case). Trans. Amer. Math. Soc., 358(3):1347-1364, 2006.

[16] Pierre Gabriel. The universal cover of a representation-finite algebra. In Representations of algebras (Puebla, 1980), volume 903 of Lecture Notes in Math., pages 68-105. Springer, Berlin, 1981.

[17] David Hughes and Josef Waschbüsch. Trivial extensions of tilted algebras. Proc. London Math. Soc. (3), 46(2):347-364, 1983.

[18] Bernhard Keller. Deformed Calabi-Yau completions. J. Reine Angew. Math., 654:125-180, 2011. With an appendix by Michel Van den Bergh.

[19] Hipolito Treffinger. $\tau$-tilting theory and $\tau$-slices. J. Algebra, 481:362$392,2017$. 
(Ibrahim Assem, Juan Carlos Bustamante) Département de Mathématiques, Université de Sherbrooke, Sherbrooke, Québec, Canada J1K2R1

E-mail address: ibrahim.assem@usherbrooke.ca juan.carlos.bustamante@usherbrooke.ca

Current address, Juan Carlos Bustamante: Mathematics Department, Champlain College, Lennoxville, 2580 Rue College, Sherbrooke, Québec, Canada J1M 2K3

(Julie Dionne)

E-mail address: dionnej@gmail.com

(Patrick Le Meur) Laboratoire de Mathématiques, Université Blaise Pascal \& CNRS, Complexe Scientifique Les Cézeaux, BP 80026, 63171 Aubière CEDEX, FRANCE

Current address, Patrick Le Meur: Univ Paris Diderot, Sorbonne Paris Cité, Institut de Mathématiques de Jussieu-Paris Rive Gauche, UMR 7586, CNRS, Sorbonne Universités, UPMC Univ Paris 06, F-75013, Paris, France

E-mail address: patrick.le-meur@imj-prg.fr

(David Smith) Department of Mathematics, Bishop's University. Sherbrooke, QuéBec, Canada, J1M1Z7

E-mail address: dsmith@ubishops.ca 